TRANSACTIONS OF THE

AMERICAN MATHEMATICAL SOCIETY

Volume 351, Number 4, April 1999, Pages 1461-1480

S 0002-9947(99)02197-2

\title{
DECOMPOSING EUCLIDEAN SPACE WITH A SMALL NUMBER OF SMOOTH SETS
}

\author{
JURIS STEPRĀNS
}

\begin{abstract}
Let the cardinal invariant $\mathfrak{s}_{n}$ denote the least number of continuously smooth $n$-dimensional surfaces into which $(n+1)$-dimensional Euclidean space can be decomposed. It will be shown to be consistent that $\mathfrak{s}_{n}$ is greater than $\mathfrak{s}_{n+1}$. These cardinals will be shown to be closely related to the invariants associated with the problem of decomposing continuous functions into differentiable ones.
\end{abstract}

\section{INTRODUCTION}

This paper is concerned with problems arising from partitioning Euclidean space into subsets with certain smoothness properties. A geometric cardinal invariant will be introduced which is determined by the least number of smooth surfaces into which Euclidean space can be partitioned and it will be shown that this invariant depends on the dimension of the Euclidean space being considered. Results obtained about these cardinal invariants will then be applied to the problem of decomposing a given continuous function into differentiable functions. The relationship established will answer a question of Morayne and Cichon who wondered whether there is a continuous function from the reals to the reals which cannot be partitioned into fewer than $2^{\aleph_{0}}$ differentiable functions. Finally, a question of Todorcevic will be answered by showing that some of the cardinal invariants introduced distinguish between the iteration and product of $\omega_{2}$ Sacks forcing partial orders.

The key interest of Theorem 5.1, which will show that it is possible to decompose Euclidean space into fewer than $2^{\aleph_{0}}$ smooth surfaces, is that smoothness is more difficult to handle than other properties of analysis. For example, it is easy to use $\sigma$-linked ${ }^{1}$ forcing to decompose any Euclidean space into $\aleph_{1}$ continuous, oneto-one and even rectifiable ${ }^{2}$ curves. To see that this is not so easily extended to differentiable curves, consider the natural forcing for decomposing the plane into rectifiable curves. It consists of triples $(c, x, \epsilon)$ where $c$ is a continuous curve of length less than $1, \epsilon>0$ and $x$ is a finite subset of the $c$. Extension is defined by $(c, x, \epsilon) \leq\left(c^{\prime}, x^{\prime}, \epsilon^{\prime}\right)$ if and only if the uniform norm of $c-c^{\prime}$ is less than $\epsilon$,

Received by the editors March 9, 1995 and, in revised form, May 5, 1997.

1991 Mathematics Subject Classification. Primary 04A30; Secondary 28A15.

Key words and phrases. Cardinal invariant, Sacks real, tangent plane, covering number.

Research for this paper was partially supported by NSERC of Canada. The author would also like to acknowledge that this paper has significantly benefitted from several remarks of A. Miller.

${ }^{1} \mathrm{~A}$ partial order is $\sigma$-linked if it is the union of countably many subsets, each of which has the property that any two of its elements have a lower bound.

${ }^{2} \mathrm{~A}$ curve is rectifiable if it has a length - in other words, the supremum of the length of piecewise linear curves obtained by connecting finite subsets on the curve exists.

(C)1999 American Mathematical Society 
$x^{\prime} \supseteq x$ and $\epsilon^{\prime} \leq \epsilon$. It is easy to see that forcing with the product of countably many copies of this partial order will cover the ground model plane with countably many rectifiable curves ${ }^{3}$. To see that the partial order is $\sigma$-linked, let $p$ be a curve of length $\delta<1$ defined by polynomials with rational coefficients. Let $\left\{A_{i}\right\}_{i \in k}$ be open balls centred at points of $p$ such that the sum of the diameters of the balls $A_{i}$ is less than $(1-\delta) / 3$. If $\left(c^{0},\left\{a_{i}^{0}\right\}_{i \in k}, \epsilon^{0}\right)$ and $\left(c^{1},\left\{a_{i}^{1}\right\}_{i \in k}, \epsilon^{1}\right)$ are such that $a_{i}^{j} \in A_{i}$, $\epsilon^{j}>4 \epsilon$ and $\left\|c^{j}-p\right\|<\epsilon$, then $\left(c^{0},\left\{a_{i}^{0}\right\}_{i \in k}, \epsilon^{0}\right)$ and $\left(c^{1},\left\{a_{i}^{1}\right\}_{i \in k}, \epsilon^{1}\right)$ are compatible.

Trying to mimic this to obtain a smooth decomposition would entail adding the derivative to the condition $(c, x, \epsilon)$ and insisting that extension now means that derivatives stay within $\epsilon$ of each other. However, this completely destroys the $\sigma$ linkedness argument because, although it is possible to insist that $a_{i}^{0}$ is close to $a_{i}^{1}$, it is not so easy to control the angle formed by these two points. This paper provides an alternate approach which allows this difficulty to be avoided.

Section 2 contains the precise definitions of the cardinal invariants discussed as well as definitions of intermediate invariants which will play a role in the proof of the main theorem. Section 3 contains lower bounds and relations between the cardinal invariants which can be proved without extra set theoretic assumptions. Section 4 contains the definition and key properties of the generalization of Sacks forcing which is used to establish later consistency results. The key lemmas that establish preservation properties of this partial order under countable support iteration are established in Section 5. From these, the main theorem follows easily. Section 6 relates the previous results to the problem of Cichon and Morayne concerning decomposing continuous functions into differentiable ones. Section 7 contains remarks about the significance of the cardinal invariants discussed here to the problem of iterating with countable support beyond $\omega_{2}$. A question of Todorcevic is also answered and some open questions are posed.

\section{BASIC CONCEPTS}

Before introducing the main concepts, some notation will be established. Let $P_{k, n}$ denote the space of all subspaces of $\mathbb{R}^{n}$ of dimension less than or equal to $k$. Define a metric $\rho$ on $P_{k, n}$ by letting $\rho(V, W)$ measure the angle between $V$ and $W$. To be precise, $\rho(V, W)$ is essentially defined to be the Hausdorff distance between $V$ and $W$ intersected with the unit sphere, or, in other words, $\rho(V, W)$ is defined to be the maximum of

$$
\left\{\max _{x \in V^{+}} \min _{y \in W^{+}} \arccos \left(\frac{x \cdot y}{\|x\|\|y\|}\right), \max _{x \in W^{+}} \min _{y \in V^{+}} \arccos \left(\frac{x \cdot y}{\|x\|\|y\|}\right)\right\}
$$

where $V^{+}$and $W^{+}$are the non-zero elements of the corresponding subspaces. If $v \in \mathbb{R}^{n}$ and $\langle v\rangle$ denotes the space spanned by $v$, then $\rho(\langle v\rangle, V)$ will be abbreviated to $\rho(v, V)$ and if $v^{\prime} \in \mathbb{R}^{n}$, then $\rho\left(\langle v\rangle,\left\langle v^{\prime}\right\rangle\right)$ will be abbreviated to $\rho\left(v, v^{\prime}\right)$.

The following definition plays a central role in this paper. It generalizes the notion of a tangent plane to the context of a set rather than a function. The case of one dimensional tangents was introduced by Besicovitch in [4] and higher dimensional analogues have been considered in [2].

\footnotetext{
${ }^{3}$ In particular, if $G$ is generic for the countable product let $\gamma_{i}$ be the uniform limit of the curves $c$ such that there is some $p \in G$ such that $p(i)=(c, x, \epsilon)$ for some $x$ and $\epsilon$. Given $w \in \mathbb{R}^{2}$ in the ground model and a condition $p$ choose a coordinate $j$ such that $p(j)=\emptyset$ and extend $p$ to $p^{\prime}$ such that $p^{\prime}(j)=(c,\{w\}, 1)$ where $c$ is an arbitrary curve containing $w$. It is then routine to check that $p^{\prime}$ forces $\gamma_{j}$ to contain $w$.
} 
Definition 2.1. If $A \subseteq \mathbb{R}^{n}, V \in P_{k, n}$ and $b \in \mathbb{R}^{n}$, then $V$ will be said to be a $k$-dimensional tangent plane to $A$ at $b$ if and only if for every $\epsilon>0$ there is some $\delta>0$ such that $\rho(V, b-a)<\epsilon$ for every $a \in A$ such that $0<\|a-b\|<\delta$. A subset $A \subseteq \mathbb{R}^{n}$ will be said to be a $k$-smooth set if for every $b \in A$ there is $V \in P_{k, n}$ which is a $k$-dimensional tangent plane ${ }^{4}$ to $A$ at $b$.

In the following discussion, there will also be occasion to refer to a continuously smooth set, by which is meant a set to each point of which it is possible to assign, in a continuous fashion, a tangent plane. A subset $A \subseteq \mathbb{R}^{n}$ will be said to be continuously- $k$-smooth if there is a continuous function $D: A \rightarrow P_{n, k}$ such that each $D(a)$ is a $k$-dimensional tangent plane to $A$ at $a$. By a $k$-smooth surface in $\mathbb{R}^{m}$ will be meant a subset of $\mathbb{R}^{m}$ which is isometric to (the graph of) some differentiable function from $\mathbb{R}^{k}$ to $\mathbb{R}^{m-k}$. It is easy to see that a $k$-smooth set need not be a $k$-smooth surface, nor even the union of finitely many $k$-smooth surfaces ${ }^{5}$. However, it will be shown that $k$-smooth sets can be covered by a sufficiently small number of $k$-smooth surfaces so that some interesting results will follow. The next two lemmas will, along with results from Section 3, establish this fact.

Lemma 2.1. Let $X \subseteq \mathbb{R}^{n}$ be a closed subset, $\hat{X} \subseteq X$ be a dense $G_{\delta}$ and $F: X \rightarrow$ $\mathbb{R}^{k}$ be a continuous function. If $D: \hat{X} \rightarrow P_{n+k, n}$ is a continuous function, then the set of points $x$ at which $D(x)$ is a tangent to the graph of $F$ can be expressed as the union of $\mathfrak{d}$ closed sets.

Proof. Using continuity, let $U \subseteq X$ be a countable dense subset and notice that for every $\epsilon>0, \delta>0$ and $u \in U$

$$
\{x \in \hat{X}:\|x-u\| \geq \delta \text { or } \rho((x, F(x))-(u, F(u)), D(x)) \leq \epsilon\}
$$

is closed in $\hat{X}$. Hence, a direct calculation using the definition of a tangent yields that the set of points $x$ at which $D(x)$ is tangent to the graph of $F$ is a relative $G_{\delta \sigma}$ in $\hat{X}$ and, hence, is itself a $G_{\delta \sigma}$. Therefore, if this set of points is $\bigcap_{n \in \omega} \bigcup_{m \in \omega} C_{m}^{n}$ where each $C_{m}^{n}$ is closed let $B_{\xi}=\bigcap_{n \in \omega} \bigcup_{m \in \xi(n)} C_{m}^{n}$ for $\xi \in \omega_{\omega}^{\omega}$ and note that each $B_{\xi}$ is closed. If $\mathcal{D}$ is any dominating family in $\omega_{\omega}$, then $\bigcup_{\xi \in \mathcal{D}} B_{\xi}$ is equal to the set of points at which the derivative of $F$ is given by $D$.

The proof of the next lemma is simplified by using the following definition.

Definition 2.2. A set $E \subseteq \mathbb{R}^{n}$ is said to be $k$-Lipschitz if there are a $k$-dimensional hyperplane $H \subseteq \mathbb{R}^{n}$ and an angle $\theta<\pi / 2$ such that $\rho(x-y, H)<\theta$ for any two points $x$ and $y$ from $E$.

Observe that any $k$-Lipschitz set can be viewed as the graph of a function from $\mathbb{R}^{k}$ to $\mathbb{R}^{n-k}$ in a suitable coordinate system. If $E \subseteq \mathbb{R}^{n}$ is $k$-Lipschitz and this is witnessed by the hyperplane $H \subseteq \mathbb{R}^{n}$, then, for each $z \in \mathbb{R}^{k}$, let $V_{z}$ be the $(n-k)$ dimensional hyperplane orthogonal to $H$ and containing $z$. It follows that $E$ can intersect $V_{z}$ on at most one point - if the intersection is not empty, then let $E^{*}(z)$

\footnotetext{
${ }^{4}$ Observe that if $A \subseteq \mathbb{R}^{n}$ and $V$ is a $k$-dimensional tangent plane to $A$ at $b$ and if there is no $m$-dimensional tangent plane to $A$ at $b$, then $V$ is the unique $k$-dimensional tangent plane to $A$ at $b$. However it is possible that a $k$-dimensional smooth set in $\mathbb{R}^{n}$ may have $m$-dimensional tangent planes at some - or even all — points, where $m<k$. In this respect, the generalization of a tangent line to a tangent plane in Definition 2.1 differs from that found on page 263 of [7].

${ }^{5}$ Consider a sequence of circles $\left\{C_{n}\right\}_{n \in \omega}$ each of which is tangent to the horizontal axis at the origin and $C_{n}$ having radius $n$.
} 
be this unique point. It is easy to see that for each $\epsilon>0$ the set of points at which the oscillation ${ }^{6}$ of $E^{*}$ is greater than $\epsilon$ must be a closed discrete set. Hence $E^{*}$ is of Baire class 1 on its domain. Indeed, since the closure of $E$ is still $k$-Lipschitz, it follows that $E^{*}$ is the restriction of a Baire class 1 function defined on all of $\mathbb{R}^{k}$.

The following theorem of Aversa, Laczkovich and Preiss [2] will be used in the next lemma.

Theorem 2.1. If $H$ is a closed subset of $\mathbb{R}^{n}, F: H \rightarrow \mathbb{R}$ and $L: H \rightarrow P_{n, n+1}$ is a derivative of $F$ on $H$, then $F$ can be extended to a function $\hat{F}$ everywhere differentiable on $\mathbb{R}^{n}$ if and only if $L$ is Baire class 1.

Lemma 2.2. If $X \subseteq \mathbb{R}^{m}$ is a continuously $k$-smooth set, then there are $\mathfrak{d} k$-smooth surfaces in $\mathbb{R}^{m}$ whose union contains $X$.

Proof. Let $X \subseteq \mathbb{R}^{m}$ be an arbitrary continuously $k$-smooth set. The proof of the first theorem of [4] can be extended to show that $X$ is contained in a countable union of sets, each of which is $k$-Lipschitz. To see this, simply let $0<\theta<\pi / 4$, let $\left\{H_{n}\right\}_{n \in \omega}$ enumerate a dense set of $k$-dimensional hyperplanes in $\mathbb{R}^{m}$ and define $L(n, j)$ to be the set of all $x \in X$ such that if $x^{\prime} \in X \backslash\{x\}$ and $\left\|x-x^{\prime}\right\|<1 / j$, then $\rho\left(x-x^{\prime}, H_{n}\right)<\theta$.

From the remarks following Definition 2.2 it follows that $X$ can be covered by countably many functions of Baire class 1 and with closed domain, each with respect to some coordinate system. It is shown in [5] that any such function can be covered using no more than $\mathfrak{d}$ continuous functions.

Let $f: \mathbb{R}^{k} \rightarrow \mathbb{R}^{m-k}$ be one of these continuous functions where it is being supposed that, without loss of generality, its coordinate system is the standard one. Let $f^{*}$ be the closure of $f \cap X$ and let $X^{*}$ be the domain of $f^{*}$. Let $D: X \rightarrow P_{m, k}$ witness that $X$ is continuously $k$-smooth and define $\hat{D}(x)=D(x, f(x))$ for any $x$ such that $(x, f(x)) \in X$. Let $\hat{X}$ be the $G_{\delta}$ consisting of all points to which $\hat{D}$ can be extended continuously and denote this extension also by $\hat{D}$. A direct computation shows that the set of all $x \in \hat{X}$ such that $\hat{D}(x)$ is a derivative to $f^{*}(x)$ is a relative $G_{\delta \sigma}$ and, since $\hat{X}$ is itself a $G_{\delta}$, this set is a real $G_{\delta \sigma}$. From Lemma 2.1, there are $\mathfrak{d}$ closed sets, $\left\{B_{\alpha}\right\}_{\alpha \in \mathfrak{d}}$ such that

$$
\{x:(x, f(x)) \in X\} \subseteq\left\{x: \hat{D}(x) \text { is a derivative to } f^{*}(x)\right\}=\bigcup_{\alpha \in \mathfrak{d}} B_{\alpha}
$$

and so $\hat{D}(x)$ is a derivative of $f^{*} \uparrow B_{\alpha}$ for each $x \in B_{\alpha}$. The continuity of $\hat{D}$ together with Theorem 2.1 ensures that $f^{*}\left\lceil B_{\alpha}\right.$ can be extended to a function which is differentiable on all of $\mathbb{R}^{k}$. Its graph is therefore a $k$-smooth surface.

Notice that if it is only desired to extend a smooth set to a Borel set, rather than a function whose domain is all of Euclidean space, then this is easy, as the following lemma demonstrates.

Lemma 2.3. If $S \subseteq \mathbb{R}^{m}$ is an $n$-smooth set, then there is a Borel set $S^{\prime} \supseteq S$ which is also an $n$-smooth set. Similarly if $S \subseteq \mathbb{R}^{m}$ is a continuously $n$-smooth set, then there is a Borel set $S^{\prime} \supseteq S$ which is also a continuously $n$-smooth set.

\footnotetext{
${ }^{6}$ The oscillation of a function $f: \mathbb{R} \rightarrow \mathbb{R}$ at a point $x$ is

$$
\lim _{\epsilon \rightarrow 0} \sup \{|f(a)-f(b)|:|a-x|,|b-x|<\epsilon\} .
$$
}


Proof. Choose $D$, a countable dense subset $S$ and let $S^{\prime}$ be the set of all those $x$ such that for every $\epsilon>0$ there exists $\delta>0$ if $d_{1}$ and $d_{2}$ belong to $D$ and $\left\|d_{1}-x\right\|$ and $\left\|d_{2}-x\right\|$ are both less than $\delta$, then $\rho\left(x-d_{1}, x-d_{2}\right)<\epsilon$. In the continuous case extend the derivative continuously to a $G_{\delta}$.

The main object of study in this paper is decompositions of Euclidean space into smooth surfaces and the next definition introduces convenient cardinal invariants with which to discuss this.

Definition 2.3. Let $\mathcal{S}_{m, n}$ denote the $\sigma$-ideal generated by the $n$-smooth surfaces in $\mathbb{R}^{m}$. As well, define $\mathcal{D}_{m, n}$ to be the $\sigma$-ideal generated by the $n$-smooth subsets of $\mathbb{R}^{m}$ and $\mathcal{C}_{m, n}^{1}$ the $\sigma$-ideal generated by the continuously $n$-smooth subsets of $\mathbb{R}^{m}$.

The covering number of an ideal $\mathcal{I}$ will be denoted by $\operatorname{cov}(\mathcal{I})$ while unif $(\mathcal{I}), \operatorname{cof}(\mathcal{I})$ and $\operatorname{add}(\mathcal{I})$ will represent the uniformity, cofinality and additivity invariants. The present paper is primarily concerned with $\operatorname{cov}\left(\mathcal{S}_{m, n}\right)$; however some remarks about the other invariants are in order. A. Miller has noticed the following identities.

Proposition 2.1. If $m>n$, then $\operatorname{cof}\left(\mathcal{D}_{m, n}\right)=\operatorname{cof}\left(\mathcal{S}_{m, n}\right)=\operatorname{cof}\left(\mathcal{C}_{m, n}^{1}\right)=2^{\aleph_{0}}$ and $\operatorname{add}\left(\mathcal{D}_{m, n}\right)=\operatorname{add}\left(\mathcal{C}_{m, n}^{1}\right)=\operatorname{add}\left(\mathcal{S}_{m, n}\right)=\omega_{1}$.

Proof. Only the case of $\mathcal{D}_{m, n}$ will be discussed since the others are similar. Both identities follow from the fact that if $\mathcal{H}$ is an uncountable family of $n$-dimensional, parallel hyperplanes in $\mathbb{R}^{m}$, then $\bigcup \mathcal{H} \notin \mathcal{D}_{m, n}$. To see that this is so, let $X \in \mathcal{D}_{m, n}$ and let $X=\bigcup_{i \in \omega} X_{i}$ where each $X_{i}$ is $n$-smooth and suppose that $\bigcup \mathcal{H} \subseteq X$. Using Lemma 2.3, it may be assumed that each $X_{i}$ has the property of Baire. For $H \in \mathcal{H}$ let $i(H) \in \omega$ and $R(H)$ be a rational, $n$-dimensional rectangle such that $X_{i(H)} \cap H$ is comeagre in $R(H)$. If $j$ is such that there is a rational, $n$-dimensional rectangle $R$ and there are uncountably many $H \in \mathcal{H}$ such that $i(H)=j$ and $R(H)=R$, then $X_{j}$ cannot be $n$-smooth.

While cof and add are not monotone invariants with respect to inclusion, the invariant unif is, and hence, it follows from the fact that $\mathcal{S}_{m, n} \cup \mathcal{C}_{m, n}^{1} \subseteq \mathcal{D}_{m, n}$ that $\operatorname{unif}\left(\mathcal{S}_{m, n}\right)+\operatorname{unif}\left(\mathcal{C}_{m, n}^{1}\right) \leq \operatorname{unif}\left(\mathcal{D}_{m, n}\right)$. However the following questions remain open.

Question 2.1. What is the value of $\operatorname{unif}\left(\mathcal{S}_{m, n}\right)$ ? What is the value of unif $\left(\mathcal{C}_{m, n}^{1}\right)$ ? What is the value of $\operatorname{unif}\left(\mathcal{D}_{m, n}\right)$ ? In particular, can any of these be greater than $\omega_{1}$ ?

Finally, some simple inequalities will be established.

Proposition 2.2. If $m \geq m^{\prime}>n$, then

- $\operatorname{cov}\left(\mathcal{D}_{m, n}\right) \geq \operatorname{cov}\left(\mathcal{D}_{m+1, n+1}\right)$,

- $\operatorname{cov}\left(\mathcal{S}_{m, n}\right) \geq \operatorname{cov}\left(\mathcal{S}_{m+1, n+1}\right)$,

- $\operatorname{cov}\left(\mathcal{C}_{m, n}^{1}\right) \geq \operatorname{cov}\left(\mathcal{C}_{m+1, n+1}^{1}\right)$,

- $\operatorname{cov}\left(\mathcal{D}_{m, n}\right) \geq \operatorname{cov}\left(\mathcal{D}_{m^{\prime}, n}\right)$,

- $\operatorname{cov}\left(\mathcal{S}_{m, n}\right) \geq \operatorname{cov}\left(\mathcal{S}_{m^{\prime}, n}\right)$,

- $\operatorname{cov}\left(\mathcal{C}_{m, n}^{1}\right) \geq \operatorname{cov}\left(\mathcal{C}_{m^{\prime}, n}^{1}\right)$,

and, moreover, $\operatorname{cov}\left(\mathcal{C}_{m, n}^{1}\right) \geq \operatorname{cov}\left(\mathcal{D}_{m, n}\right) \geq \omega_{1}$ and $\operatorname{cov}\left(\mathcal{S}_{m, n}\right) \geq \operatorname{cov}\left(\mathcal{D}_{m, n}\right) \geq \omega_{1}$.

Proof. The proofs for each of the ideals are the same so only the smooth case will be considered. For the first inequality let $\left\{S_{\xi}\right\}_{\xi \in \kappa}$ be $n$-smooth sets in $\mathbb{R}^{m}$ and note 
that $S_{\xi} \times \mathbb{R}$ is $(n+1)$-smooth in $\mathbb{R}^{m+1}$. For the second inequality, take any cover of $\mathbb{R}^{m}$ by $n$-smooth sets and intersect each member with a fixed subspace of dimension $m^{\prime}$. The first two inequalities of the last clause are obvious and the last inequality follows from observing that $\mathcal{D}_{m, n}$ consists of meagre sets because $m>n$.

\section{Absolute LOWer Bounds}

This section will provide lower bounds for $\operatorname{cov}\left(\mathcal{D}_{m, n}\right)$ which, in light of Proposition 2.2, provide lower bounds for $\operatorname{cov}\left(\mathcal{S}_{m, n}\right)$ and $\operatorname{cov}\left(\mathcal{C}_{m, n}^{1}\right)$ as well. For the sake of the next theorem, let $\mathcal{D}_{m, 0}$ denote the $\sigma$-ideal generated by the discrete subsets of $\mathbb{R}^{m}$ and note that $\operatorname{cov}\left(\mathcal{D}_{m, 0}\right)=2^{\aleph_{0}}$. Also, $\mathcal{D}_{m, n}^{\lambda}$ will denote the ideal generated by unions of subsets of $\mathcal{D}_{m, n}$ of cardinality less than $\lambda$.

Theorem 3.1. If $n^{\prime}+k^{\prime}=m, k \leq k^{\prime}, n \leq n^{\prime}$ and $k+n=j+1$

$$
\operatorname{unif}\left(\mathcal{D}_{k^{\prime}, k-1}^{\lambda}\right) \cdot \operatorname{cov}\left(\mathcal{D}_{m, j}\right)<\operatorname{cov}\left(\mathcal{D}_{n^{\prime}, n-1}\right),
$$

then $\operatorname{cov}\left(\mathcal{D}_{m, j}\right) \geq \lambda$.

Proof. Let $\operatorname{cov}\left(\mathcal{D}_{m, j}\right)=\kappa$ and let $\left\{S_{\alpha}\right\}_{\alpha \in \kappa}$ be $j$-smooth sets such that $\bigcup_{\alpha \in \kappa} S_{\alpha}=$ $\mathbb{R}^{m}$. Since $m=n^{\prime}+k^{\prime}$, it is possible to let $V \subseteq \mathbb{R}^{m}$ be an $n^{\prime}$-dimensional subspace and let $W$ be a $k^{\prime}$-dimensional subspace orthogonal to $V$. Letting $\mu=\operatorname{unif}\left(\mathcal{D}_{k^{\prime}, k-1}^{\lambda}\right)$, let $B=\left\{w_{\xi}\right\}_{\xi \in \mu}$ be a subset of $W$ which cannot be decomposed into less than $\lambda$ elements of $\mathcal{D}_{k^{\prime}, k-1}$. For each $\xi \in \kappa$ let $S_{\xi, \alpha}$ consist of all those $s \in S_{\alpha} \cap\left(V+w_{\xi}\right)$ such that if $T$ is a tangent plane ${ }^{7}$ to $S_{\alpha} \cap\left(V+w_{\xi}\right)$ at $s$, then $T / T \cap V$ has dimension at least $k$. Since $n+k=j+1$ and $S_{\alpha}$ is $j$-smooth, it follows that for each $s \in S_{\xi, \alpha}$ the plane tangent to $S_{\alpha}$ at $s$ intersects $V$ on a subspace of dimension smaller than $n$ and so $S_{\xi, \alpha}$, considered as subset of $V$ under the obvious isomorphism, is a $(n-1)$ smooth set. Since $\kappa \cdot \mu<\operatorname{cov}\left(\mathcal{D}_{n^{\prime}, n-1}\right)$, it follows that there is some $v \in V$ such that $v+w_{\xi} \notin S_{\xi, \alpha}$ for all $\xi \in \mu$ and $\alpha \in \kappa$.

Then $B=\bigcup_{\alpha \in \kappa} C_{\alpha}$ where $C_{\alpha}=\left\{w \in B: v+w \in S_{\alpha}\right\}$. If $\kappa<\lambda$, it follows that there is $\alpha \in \kappa$ such that $C=\{v+w\}_{w \in C_{\alpha}}$ does not belong to $\mathcal{D}_{k^{\prime}, k-1}$. This means that that there is a point $v+w_{\zeta} \in C$ such that $C$ has no $(k-1)$-dimensional tangent plane at $v+w_{\zeta}$. However, $S_{\alpha}$ does have a $j$-dimensional tangent plane, $T$, at $v+w_{\zeta}$. The definition of $S_{\zeta, \alpha}$ implies that the dimension of $T / V \cap T$ is less than $k$. This contradicts the fact that $T / V \cap T$ is a tangent to $C$ at $v+w_{\zeta}$ and, hence, shows that $\operatorname{cov}\left(\mathcal{D}_{m, j}\right)=\kappa \geq \lambda$.

Corollary 3.1. If $m>n \geq 1$, then $\operatorname{cov}\left(\mathcal{D}_{m, n}\right)^{+} \geq \operatorname{cov}\left(\mathcal{D}_{m-1, n-1}\right)$.

Proof. If $\operatorname{cov}\left(\mathcal{D}_{m, n}\right) \geq \operatorname{cov}\left(\mathcal{D}_{m-1, n-1}\right)$, then there is nothing to prove. Otherwise, $\operatorname{cov}\left(\mathcal{D}_{m, n}\right)<\operatorname{cov}\left(\mathcal{D}_{m-1, n-1}\right)$ and so, if $\lambda<\operatorname{cov}\left(\mathcal{D}_{m-1, n-1}\right)$, then

$$
\operatorname{unif}\left(\mathcal{D}_{1,0}^{\lambda}\right) \cdot \operatorname{cov}\left(\mathcal{D}_{m, n}\right)<\operatorname{cov}\left(\mathcal{D}_{m-1, n-1}\right)
$$

and so it follows from Theorem 3.1 that $\operatorname{cov}\left(\mathcal{D}_{m, n}\right) \geq \lambda$. Since $\lambda<\operatorname{cov}\left(\mathcal{D}_{m-1, n-1}\right)$ was arbitrary, it follows that

$$
\operatorname{cov}\left(\mathcal{D}_{m, n}\right)^{+} \geq \operatorname{cov}\left(\mathcal{D}_{m-1, n-1}\right)
$$

${ }^{7}$ Recall that tangent planes have been defined as subspaces so it does not make sense to say, as would be natural, that $V+w_{\xi}$ does not contain a tangent plane to $S_{\alpha}$ at $s$. 
Note that from Proposition 2.2 and Corollary 3.1 it follows that

$$
\operatorname{cov}\left(\mathcal{D}_{m, n}\right)^{+} \geq \operatorname{cov}\left(\mathcal{D}_{m-1, n-1}\right) \geq \operatorname{cov}\left(\mathcal{D}_{m, n}\right)
$$

provided that $m>n$.

Corollary 3.2. If $m \geq 2$, then $\operatorname{cov}\left(\mathcal{D}_{m, 1}\right)^{+} \geq 2^{\aleph_{0}}$.

Proof. Let $n=1$ in Corollary 3.1 and note that $\operatorname{cov}\left(\mathcal{D}_{m-1,0}\right)=2^{\aleph_{0}}$ provided that $m \geq 2$.

It is worth noting that if $k<n$ and $S \subseteq \mathbb{R}^{n}$ is $k$-smooth, then $S$ is nowhere dense. Hence $\operatorname{cov}\left(\mathcal{D}_{m, n}\right) \geq \operatorname{cov}(\mathcal{M})$ where $\mathcal{M}$ denotes the meagre ideal. Moreover, it will be shown in Theorem 6.1 that $\operatorname{cov}\left(\mathcal{D}_{2,1}\right) \geq \operatorname{cov}(\mathcal{N})$ where $\mathcal{N}$ is the ideal of null sets with respect to Lebesgue measure, but this is also a consequence of the next proposition. The proof of this proposition will require some preliminary definitions.

Definition 3.1. Given an integer $n \geq 1,1 \leq j \leq n$ and $q \in \mathbb{Q}$ let $H_{q, j}^{n} \subseteq \mathbb{R}^{n}$ be the $(n-1)$-dimensional hyperplane which is orthogonal to the $j^{\text {th }}$ coordinate axis and intersects this axis at $q$. A subset $X \subseteq \mathbb{R}^{n} \backslash \mathbb{Q}^{n}$ will be said to be very nowhere dense if for each $q \in \mathbb{Q}$ and each $1 \leq j \leq n$ no point $x \in H_{q, j}^{n}$ is in the closure of $X$ from both sides of the hyperplane $H_{q, j}^{n}$. The cardinal invariant $\mathfrak{d}_{n}$ is defined to be the covering invariant ${ }^{8}$ of the ideal generated by the very nowhere dense subsets of $\mathbb{R}^{n}$.

Since closed subsets of the irrationals are clearly very nowhere dense and it is well known that $\mathfrak{d}$ is equal to the least number of closed sets of irrationals required to cover the irrationals, it follows that $\mathfrak{d}_{1} \leq \mathfrak{d}$. Moreover, if $A_{1}$ and $A_{2}$ are very nowhere dense subsets of $\mathbb{R}^{n_{1}}$ and $\mathbb{R}^{n_{2}}$, then $A_{1} \times A_{2}$ is a very nowhere dense subset of $\mathbb{R}^{n_{1}} \times \mathbb{R}^{n_{2}}$. Notice as well that the Baire Category Theorem implies that $\mathfrak{d}_{n}$ cannot be countable. This establishes the following.

Proposition 3.1. If $n \geq 1$, then $\omega_{1} \leq \mathfrak{d}_{n} \leq \mathfrak{d}_{1} \leq \mathfrak{d}$.

Proposition 3.2. If $n \geq 1$, then $\operatorname{cov}\left(\mathcal{S}_{n+1, n}\right) \geq \mathfrak{d}_{n}$.

Proof. Let $\left\{p_{n}\right\}_{n \in \omega}$ enumerate $\mathbb{Q}^{n}$. Let

$$
L\left(x_{1}, x_{2}, \ldots, x_{n}\right)=\sum\left\{\frac{1}{2^{i}}: p_{i}=\left(q_{1}, q_{2}, \ldots, q_{n}\right) \text { and }(\forall j \leq n)\left(q_{j}<x_{j}\right)\right\}
$$

and define

$$
H\left(x_{1}, x_{2}, \ldots, x_{n}\right)=\int_{-\infty}^{x_{1}} \int_{-\infty}^{x_{2}} \ldots, \int_{-\infty}^{x_{n}} L\left(t_{1}, t_{2}, \ldots, t_{n}\right) d t_{1} d t_{2} \ldots d t_{n} .
$$

Now, if $\left\{X_{\alpha}\right\}_{\alpha \in \kappa}$ are $n$-smooth surfaces in $\mathbb{R}^{n+1}$, then the projection onto $\mathbb{R}^{n}$ of each set $X_{\alpha} \cap H$ must be a very nowhere dense set in $\mathbb{R}^{n}$. To see this, fix $\alpha \in \kappa$ and suppose that $1 \leq j \leq n, q \in \mathbb{Q}$ and $\bar{x}=\left(x_{1}, x_{2}, \ldots, x_{j-1}, q, \ldots, x_{n}\right)$ belongs to the hyperplane which intersects the $j^{\text {th }}$ axis at $q$ and is parallel to the other axes. Suppose also that

$$
\lim _{k \rightarrow \infty}\left(w_{1}^{k}, w_{2}^{k}, \ldots, w_{n}^{k}\right)=\lim _{k \rightarrow \infty}\left(z_{1}^{k}, z_{2}^{k}, \ldots, z_{n}^{k}\right)=\bar{x}
$$

\footnotetext{
${ }^{8}$ For more details on this see Definition 2.3 and Lemma 3.1 of [11] where "very nowhere dense" is misleadingly called "almost nowhere dense".
} 
are sequences such that $w_{j}^{k}<q<z_{j}^{k}$ for each $k \in \omega$ and that $\left(w_{1}^{k}, w_{2}^{k}, \ldots, w_{n}^{k}\right)$ and $\left(z_{1}^{k}, z_{2}^{k}, \ldots, z_{n}^{k}\right)$ belong to the projection of $X_{\alpha}$ for each $k \in \omega$.

Then

$$
\begin{aligned}
& \lim _{k \rightarrow \infty} \frac{H\left(w_{1}^{k}, w_{2}^{k}, \ldots, w_{n}^{k}\right)-H\left(x_{1}, x_{2}, \ldots, x_{n}\right)}{w^{k}-q} \\
& <\lim _{k \rightarrow \infty} \frac{H\left(z_{1}^{k}, z_{2}^{k}, \ldots, z_{n}^{k}\right)-H\left(x_{1}, x_{2}, \ldots, x_{n}\right)}{z^{k}-q}
\end{aligned}
$$

contradicting that $\bar{x} \in X_{\alpha}$ and $X_{\alpha}$ has a tangent plane at $\bar{x}$.

Before continuing, it will be noted that the cardinal invariant $\mathfrak{d}_{n}$ is even more closely related to the function $H$ of the proof of Proposition 3.2 than is suggested in that proof. In fact, the least number of smooth functions required to cover $H$ is precisely $\mathfrak{d}_{n}$. The proof of Proposition 3.2 already shows that at least $\mathfrak{d}_{n}$ smooth functions are required, but appealing to Theorem 2.1 shows that this number also suffices.

Proposition 3.3. If $m \geq n+1$, then $\operatorname{cov}\left(\mathcal{S}_{m, n}\right)=\operatorname{cov}\left(\mathcal{S}_{n+1, n}\right)$.

Proof. From Proposition 2.2 it follows that it suffices to show that $\operatorname{cov}\left(\mathcal{S}_{m, n}\right)$ is not greater than $\operatorname{cov}\left(\mathcal{S}_{n+1, n}\right)$. Proceed by induction on $m$ and assume that $m>n+1$. Let $\left\{f_{\alpha}: \mathbb{R}^{n} \rightarrow \mathbb{R}^{m}\right\}_{\alpha \in \kappa}$ be smooth parametrizations of smooth surfaces whose union covers $\mathbb{R}^{m}$. Let $f_{\alpha}(x)=\left(f_{\alpha}^{1}(x), f_{\alpha}^{2}(x), \ldots f_{\alpha}^{n}(x)\right)$ and define

$$
H_{\alpha, \beta}(x)=\left(f_{\alpha}\left(f_{\beta}^{1}(x), f_{\beta}^{2}(x), \ldots f_{\beta}^{n}(x)\right), f_{\beta}^{n+1}(x)\right)
$$

and note that each $H_{\alpha, \beta}$ is $\operatorname{smooth}^{9}$. If $(z, w) \in \mathbb{R}^{m} \times \mathbb{R}$, then there is some $z^{\prime} \in \mathbb{R}^{n}$ and $\alpha \in \kappa$ such that $f_{\alpha}\left(z^{\prime}\right)=z$. Choose $\beta \in \kappa$ and $x$ such that $f_{\beta}(x)=\left(z^{\prime}, w, t\right)$ for some $t$. Then $H_{\alpha, \beta}(x)=(z, w)$.

Proposition 3.3 provides the justification for defining $\mathfrak{s}_{n}=\operatorname{cov}\left(\mathcal{S}_{n+1, n}\right)$. From Lemma 2.2 it follows that $\operatorname{cov}\left(\mathcal{S}_{m, n}\right) \leq \operatorname{cov}\left(\mathcal{C}_{m, n}^{1}\right) \cdot \mathfrak{d}$ and this immediately implies the following.

Corollary 3.3. If $\mathfrak{d}_{n}=\mathfrak{d}$, then $\mathfrak{s}_{n} \leq \operatorname{cov}\left(\mathcal{C}_{m, n}^{1}\right) \cdot \mathfrak{d}$.

It may also be noted that from the same hypothesis as that of Corollary 3.3 it can almost be concluded that $\mathfrak{s}_{n} \in\left\{\operatorname{cov}\left(\mathcal{C}_{m, n}^{1}\right), \mathfrak{d}\right\}$. It has already been observed that $\mathfrak{s}_{n} \leq \operatorname{cov}\left(\mathcal{C}_{m, n}^{1}\right) \cdot \mathfrak{d}$. On the other hand, from Proposition 3.2 and the hypothesis it follows that $\mathfrak{s}_{n} \geq \mathfrak{d}_{n}=\mathfrak{d}$. Hence, $\mathfrak{d} \leq \mathfrak{s}_{n} \leq \operatorname{cov}\left(\mathcal{C}_{m, n}^{1}\right) \cdot \mathfrak{d}$. There are two cases to consider. If $\operatorname{cov}\left(\mathcal{C}_{m, n}^{1}\right) \leq \mathfrak{d}$, then

$$
\mathfrak{d} \leq \mathfrak{s}_{n} \leq \operatorname{cov}\left(\mathcal{C}_{m, n}^{1}\right) \cdot \mathfrak{d}=\mathfrak{d}
$$

and so $\mathfrak{s}_{n}=\mathfrak{d}$. Otherwise, $\operatorname{cov}\left(\mathcal{C}_{m, n}^{1}\right)>\mathfrak{d}$ and so

$$
\mathfrak{s}_{n} \leq \operatorname{cov}\left(\mathcal{C}_{m, n}^{1}\right) \cdot \mathfrak{d}=\operatorname{cov}\left(\mathcal{C}_{m, n}^{1}\right)
$$

and so, if $\operatorname{cov}\left(\mathcal{C}_{m, n}^{1}\right) \leq \mathfrak{s}_{n}$, it follows that $\mathfrak{s}_{n}=\operatorname{cov}\left(\mathcal{C}_{m, n}^{1}\right)$. However, the gap in this argument is still an open question.

Question 3.1. Is the inequality $\operatorname{cov}\left(\mathcal{C}_{m, n}^{1}\right) \leq \mathfrak{s}_{n}$ true?

\footnotetext{
${ }^{9}$ However, it may not be the parametrization of a function with respect to any coordinate axes; but this is easily dealt with by decomposing it into a finite number of pieces using the implicit function theorem.
} 


\section{FORCING A LOWER BOUND}

Sacks forcing will be denoted by $\mathfrak{S}$. The following definition generalizes Sacks forcing.

Definition 4.1. By $\mathfrak{S}(n)$ will be denoted the partial order, under inclusion, of all subtrees $T \subseteq{ }^{\omega}(n+1)$ such that

- for every $t \in T$ the cardinality of the set of immediate successors of $t$ in $T$ does not exceed $n+1$;

- for every $t \in T$ there is some $t^{\prime} \supseteq t$ such that $t^{\prime}$ has $n+1$ immediate successors in $T$ - such a $t^{\prime}$ is known as a branching node.

For each $n \in \omega$ choose $\epsilon(n)>0, \delta(n)>0$ and unit vectors $\left\{\vec{x}_{i}\right\}_{i=0}^{n} \subseteq \mathbb{R}^{n}$ such that, if $B_{i}$ is the ball of radius $\delta(n)$ and centre $\vec{x}_{i}$, then

$$
\begin{aligned}
& \text { if } V \subseteq \mathbb{R}^{n} \text { is a }(n-1) \text {-dimensional hyperplane and } y \in B_{j} \cap V, \\
& \text { then there exists } i \leq n \text { such that } \rho(V, y-z)>\epsilon(n) \text { for all } z \in B_{i} .
\end{aligned}
$$

Let $\left\{\nu_{n}\right\}$ be a sequence of positive reals such that if $\sum_{i=k+1}^{\infty} \nu_{i}<\nu_{k} \delta(n)$. Next, define a mapping $\Phi_{n}:(n+1)^{\omega} \rightarrow \mathbb{R}^{n}$ by

$$
\Phi_{n}(f)=\sum_{i \in \omega} \nu_{i} \vec{x}_{f(i)}
$$

and observe that $\Phi_{n}$ is one-to-one and continuous. It follows that $\Phi_{n}(\bar{T})$ is a compact subset of $\mathbb{R}^{n}$ homeomorphic to $\bar{T}$ for any $T \in \mathfrak{S}(n)$.

Lemma 4.1. If $T \in \mathfrak{S}(n)$ and $S$ is a $(n-1)$-dimensional smooth set in $\mathbb{R}^{m}$ for $m \geq n$, then $S \cap \Phi_{n}(\bar{T})$ is meagre relative to $\Phi_{n}(\bar{T})$.

Proof. Let $\mathbb{R}^{n}$ be embedded in $\mathbb{R}^{m}$ in the natural way. By Lemma 2.3 it may as well be assumed that $S$ is Borel and, hence, satisfies the Property of Baire. If $S$ is not meagre relative to $\Phi_{n}(\bar{T})$, then let $U$ be an open set in $\Phi_{n}(\bar{T})$ such that $\Phi_{n}(\bar{T}) \cap U \cap S$ is comeagre in $\Phi_{n}(\bar{T}) \cap U$. Choose $b \in \bar{T}$ such that $\Phi_{n}(b) \in U \cap S$ and let $V$ be a $(n-1)$-dimensional tangent plane to $S$ at $\Phi_{n}(b)$ and let $\delta$ be such that $\rho\left(V, a-\Phi_{n}(b)\right)<\epsilon(n)$ for every $a \in A$ such that $0<\left|a-\Phi_{n}(b)\right|<\delta$.

Let $j \in \omega$ be such that $\Phi_{n}\left(\left\{b^{\prime} \in \bar{T}: b^{\prime}\lceil j=b\lceil j\})\right.\right.$ is contained in the intersection of $U$ with the ball of radius $\delta$ around $\Phi_{n}(b)$. Let $k>j$ be such that $b \uparrow k$ has $n+1$ immediate successors $s_{0}, s_{1}, s_{2}, \ldots, s_{n}$. Let $s$ be the common root of $s_{0}, s_{1}, s_{2}, \ldots, s_{n}$ and let $W=\sum_{i=0}^{k} \nu_{i} \vec{x}_{f(i)}$. Then the image under $\Phi_{n}$ of $\left\{b^{\prime} \in \bar{T}: s_{i} \subseteq b^{\prime}\right\}$ is contained in $\nu_{k} B_{i}+W$ for $i \leq n$. Let $i$ be such that $b \supseteq s_{i}$ and use the choice of $\epsilon(n)$ and the $\left\{\nu_{m}\right\}_{m \in \omega}$ to find $j$ such that $\rho\left(V, \Phi_{n}(b)-z\right)>\epsilon(n)$ for any $z \in \nu_{k} B_{j}+W$. Choosing $z \in\left(\nu_{k} B_{j}+W\right) \cap S$ is possible because $S$ is comeagre in $U$ and $B_{j, k} \subseteq U$. This is a contradiction.

Corollary 4.1. If $g \in(n+1)^{\omega}$ is the generic real added by forcing with $\mathfrak{S}(n)$, then $\Phi_{n}(g)$ does not belong to any n-1-smooth set with a Borel code in the ground model.

Proof. Suppose that $S$ is a $n-1$-smooth set and $T \in \mathfrak{S}(n+1)$ is such that

$$
T \Vdash_{\mathfrak{S}(n+1)} \text { " } \Phi_{n}(g) \in S " .
$$

From Corollary 4.1 it follows that $\bar{T} \backslash \Phi_{n}^{-1}(S)$ is a dense $G_{\delta}$ relative to $\bar{T}$. It is then standard to find $T^{\prime} \subseteq T$ such that $T^{\prime} \in \mathfrak{S}(n+1)$ and $T^{\prime} \cap \Phi_{n}^{-1}(S)=\emptyset$. This immediately yields a contradiction since $T^{\prime} \Vdash_{\mathfrak{S}(n+1)}$ " $\Phi_{n}(g) \notin S "$. 


\section{THE ITERATION AND UPPER BOUNDS}

The countable support iteration of length $\alpha$ of forcings $\mathfrak{S}(n)$ will be denoted by $\mathbb{P}_{\alpha}^{n}$. As a convenience, by countable support will be meant that if $p \in \mathbb{P}_{\alpha}^{n}$, then $p(\gamma)={ }^{\omega}(n+1)$ for all but countably many $\gamma \in \alpha$. From Corollary 4.1 of the previous section it follows that if $V$ is a model of the Generalized Continuum Hypothesis and $G$ is $\mathbb{P}_{\omega_{2}}^{n}$ generic over $V$, then $\operatorname{cov}\left(\mathcal{D}_{m, n-1}\right)=\omega_{2}$ in $V[G]$. It will be shown in this section that $\operatorname{cov}\left(\mathcal{C}_{m, n}^{1}\right)=\omega_{1}$ in $V[G]$.

To this end, suppose that $V$ is a model of the Continuum Hypothesis, $p^{*} \in \mathbb{P}_{\omega_{2}}^{n}$ and that $p^{*} \Vdash_{\mathbb{P}_{\omega_{2}}^{n}}$ " $x \in \mathbb{R}^{m}$ ". It follows that there must be some $\alpha \in \omega_{2}$ such that $p^{*} \vdash_{\mathbb{P}_{\alpha}^{n}}$ " $x \in V\left[G \cap \mathbb{P}_{\alpha}^{n}\right]$ " and $p^{*} \Vdash_{\mathbb{P}_{\alpha}^{n}}$ " $x \notin V\left[G \cap \mathbb{P}_{\beta}^{n}\right]$ " for all $\beta \in \alpha$. It must be shown that there is, in the ground model, a continuously $n$-smooth set $S \subseteq \mathbb{R}^{m}$ as well as a condition $q \leq p^{*}$ such that $q \Vdash_{\mathbb{P}_{\omega_{2}}^{n}}$ " $x \in S$ ".

Fix $x$ and let $\mathfrak{M} \prec H\left(\aleph_{3}\right)$ be a countable elementary submodel containing $p^{*}$, $x$ and $\alpha$. Notice that if $p$ and $q$ belong to $\mathbb{P}_{\alpha}^{n} \cap \mathfrak{M}$, then $p \wedge q$ also belongs to $\mathfrak{M}$. The exact definition of $p \wedge q$ is not important here, only that it is definable in $\mathfrak{M}$. Observe that $p \wedge q$ exists in $\mathbb{P}_{\alpha}^{n}$ because the meet of each factor of the iteration is simply intersection.

Let $\mathcal{P}\left(\mathbb{P}_{\alpha}^{n} \cap \mathfrak{M}\right)$ be given the Tychonoff product topology — in other words, it is homeomorphic to the Cantor set. Let $\mathcal{F}$ be the set of all prime filters on $\mathbb{P}_{\alpha}^{n} \cap \mathfrak{M}$ - in other words, $F \in \mathcal{P}\left(\mathbb{P}_{\alpha}^{n} \cap \mathfrak{M}\right)$ such that

- if $p \in F$ and $q \geq p$, then $q \in F$;

- if $p \in F$ and $q \in F$, then $p \wedge q \in F$ and $p \wedge q \neq \emptyset$;

- if $p \in F,\left\{q_{i}\right\}_{i \in k} \subseteq \mathbb{P}_{\alpha}^{n}$ and there does not exist any $r \in \mathbb{P}_{\alpha}^{n}$ such that $r \leq p$ and $r \wedge q_{i}=\emptyset$ for each $i \in k$, then $F \cap\left\{q_{i}\right\}_{i \in k} \neq \emptyset$.

Notice that $\mathcal{F}$ is a closed set in $\mathcal{P}\left(\mathbb{P}_{\alpha}^{n} \cap \mathfrak{M}\right)$. Next, let $\mathcal{G}$ be the set of all $F \in \mathcal{F}$ such that $F$ is $\mathbb{P}_{\alpha}^{n}$ generic over $\mathfrak{M}$ and $p^{*} \in F$. To see that $\mathcal{G}$ is a dense $G_{\delta}$ relative to $\mathcal{F}$, it suffices to show that if $D$ is a dense subset of $\mathbb{P}_{\alpha}^{n} \cap \mathfrak{M}$, then $\{F \in \mathcal{F}: F \cap D \neq \emptyset\}$ is a dense open set in $\mathcal{F}$. In order to verify this, let $\mathcal{V}$ be an open set in $\mathcal{F}$. It may be assumed that

$$
\mathcal{V}=\left\{F \in \mathcal{F}: p \in F \text { and }\left\{q_{i}\right\}_{i \in k} \cap F=\emptyset\right\}
$$

for some $p \in \mathbb{P}_{\alpha}^{n} \cap \mathfrak{M}$ and $\left\{q_{i}\right\}_{i \in k} \subseteq \mathbb{P}_{\alpha}^{n} \cap \mathfrak{M}$. Because $\mathcal{V}$ is a non-empty open set in $\mathcal{F}$, it follows from elementarity that there is some $r \in \mathbb{P}_{\alpha}^{n} \cap \mathfrak{M}$ such that $r \leq p$ and $r \wedge q_{i}=\emptyset$ for each $i \in k$. Let $r^{\prime} \in D \cap \mathfrak{M}$ be such that $r^{\prime} \leq r$ and observe that $\left\{F \in \mathcal{F}: r^{\prime} \in F\right\} \subseteq \mathcal{V}$. Hence $\mathcal{G}$ is a dense $G_{\delta}$ in a closed subspace of $\mathcal{P}\left(\mathbb{P}_{\alpha}^{n}\right)$. It is worth noting that the third condition in the definition of $\mathcal{F}$ implies that $\mathcal{G}$ has a base consisting of sets of the form $\mathcal{U}(p)=\{F \in \mathcal{G}: p \in F\}$.

Let $\Psi: \mathcal{G} \mapsto \mathbb{R}^{m}$ be the mapping defined by $\mathfrak{M}[G] " \models x^{G}=\Psi(G)$ " where $x^{G}$ is the interpretation of the name $x$ in $\mathfrak{M}[G]$. Notice that $\Psi$ is continuous.

Before continuing, some notation concerning fusion arguments will be established. Let $\left\{f_{i}\right\}_{i \in \omega}$ be a sequence of finite functions satisfying the following properties:

- $f_{i}: \alpha \cap \mathfrak{M} \rightarrow \omega$

- for each $i \in \omega$ there exists a unique ordinal $\beta(i) \in \alpha \cap \mathfrak{M}$ such that $f_{i+1}(\gamma)=$ $f_{i}(\gamma)$ unless $\gamma=\beta(i)$ and $f_{i+1}(\beta(i))=f_{i}(\beta(i))+1$ where $f_{i}(\beta(i))$ is defined to be 0 if $\beta(i)$ is not in the domain of $f_{i}$;

- $\sup _{i \in \omega} f_{i}(\gamma)=\omega$ for each $\gamma \in \alpha \cap \mathfrak{M}$. 
Let $\mathfrak{S}^{*}(n)$ be the set of all $T \in \mathfrak{S}(n)$ with the additional property that for every $t \in T$ the cardinality of the set of immediate successors of $t$ in $T$ is either 1 or $n+1$. Applying a recursion argument to thin down an arbitrary condition in $\mathfrak{S}(n)$ shows that $\mathfrak{S}^{*}(n)$ is dense in $\mathfrak{S}(n)$. For $p \in \mathfrak{S}^{*}(n)$ let $I_{p}:{ }^{\omega}(n+1) \rightarrow p$ be the unique lexicographic order preserving mapping from ${ }^{\omega}(n+1)$ onto the branching nodes of $p$. For $p \in \mathfrak{S}^{*}(n)$ and $t \in{ }^{\omega}(n+1)$ let $p\langle t\rangle=\left\{s \in T: s \subseteq I_{p}(t)\right.$ or $\left.I_{p}(t) \subseteq s\right\}$.

Suppose that $r \in \mathbb{P}_{\alpha}^{n}, \Gamma \in[\alpha \cap \mathfrak{M}]<\aleph_{0}$ and $h: \Gamma \rightarrow{ }^{\omega}(n+1)$. Then $r[h]$ denotes the condition defined by

$$
r[h](\gamma)= \begin{cases}r(\gamma) & \text { if } \gamma \notin \Gamma \\ r(\gamma)\langle h(\gamma)\rangle & \text { if } \gamma \in \Gamma .\end{cases}
$$

For $p \in \mathbb{P}_{\alpha}^{n}$ the notion of what it means for $p$ to be determined is similar to that in [3]. In particular, let $\Gamma$ be the support of $f$ and define a condition $p \in \mathbb{P}_{\alpha}^{n}$ to be $f$-determined if

- $p\left\lceil\gamma \Vdash\right.$ " $p(\gamma) \in \mathfrak{S}^{*}(n)$ " for every $\gamma \in \Gamma$;

- for every $h \in \prod_{\gamma \in \Gamma}{ }^{f(\gamma)}(n+1)$ the condition $p[h \uparrow \gamma]$ decides the value of $I_{p(\gamma)}(h(\gamma))$ for each $\gamma \in \Gamma$.

By a fusion sequence will be meant a sequence of conditions $\left\{p_{i}\right\}_{i \in \omega} \subseteq \mathfrak{M}$ such that each $p_{i}$ is $f_{i}$-determined and such that for each $j \leq i$ and $h \in \prod_{\gamma \in \Gamma_{j}} f_{j}(\gamma)(n+1)$ and $\gamma \in \Gamma_{j}$ the condition $p_{i+1}\left[h\lceil\gamma]\right.$ forces $I_{p_{i}(\gamma)}(h(\gamma))$ and $I_{p_{j}(\gamma)}(h(\gamma))$ to have the same value. If $G \in \mathcal{G}$, define $H_{i}(G)$ to be the unique member of $\prod_{\gamma \in \Gamma_{i}}{ }^{f_{i}(\gamma)}(n+1)$ such that $p_{i}\left[H_{i}(G)\right] \in G$ provided that such a maximal member exists at all. Of course, $H_{i}(G)$ is only defined in the context of a given fusion sequence but, since this will always be clear, it will not be added to the notation.

Two facts are worth noting. First, it is easy to verify that the fusion of such a sequence is in $\mathbb{P}_{\alpha}^{n}$ and its domain is $\mathfrak{M} \cap \omega_{2}$. Second, if $G$ and $G^{\prime}$ are in $\mathcal{G}$ and $H_{i}(G)$ and $H_{i}\left(G^{\prime}\right)$ are defined and equal for all $i \in \omega$, then $G=G^{\prime}$.

Two cases will now be considered depending on whether or not $\alpha$ is a limit ordinal.

Lemma 5.1. If $\alpha$ is limit ordinal, then there are a continuously 1-smooth set in $\mathbb{R}^{m}, L$, and a condition $r \leq p^{*}$ such that $r \Vdash_{\mathbb{P}_{\alpha}^{n}}$ " $x \in L "$.

Proof. For each $G \in \mathcal{G}$ let $\tau(G)$ be the $\operatorname{set}^{10}$ of all $V \in P_{1, m}$ such that for all $\epsilon>0$, $q \in G$ and $\beta \in \alpha$ there is $G^{\prime} \in \mathcal{G}$ such that $G^{\prime} \cap \mathbb{P}_{\beta}^{n}=G \cap \mathbb{P}_{\beta}^{n}, q \in G^{\prime}, \Psi(G) \neq \Psi\left(G^{\prime}\right)$ and $\rho\left(V, \Psi(G)-\Psi\left(G^{\prime}\right)\right)<\epsilon$. The first thing to observe is that if $G \in \mathcal{G}$, then $\tau(G) \neq \emptyset$. To see this, first observe that $\tau(G)=\bigcap_{\beta \in \alpha \cap \mathfrak{M}} \bigcap_{q \in G} \overline{\tau(G, \beta, q)}$ where $\tau(G, \beta, q)$ is defined to be

$$
\left\{\left\langle\Psi(G)-\Psi\left(G^{\prime}\right)\right\rangle: G^{\prime} \cap \mathbb{P}_{\beta}^{n}=G \cap \mathbb{P}_{\beta}^{n}, q \in G^{\prime} \text { and } \Psi(G) \neq \Psi\left(G^{\prime}\right)\right\}
$$

and, since $P_{1, m}$ is compact and the family $\{\tau(G, \beta, q)\}_{\beta \in \alpha, q \in G}$ is centred, it suffices to show that if $\beta \in \alpha \cap \mathfrak{M}$ and $q \in G$, then $\tau(G, \beta, q) \neq \emptyset$. Since $p^{*} \vdash_{\mathbb{P}_{\alpha}^{n}}$ " $x \notin$ $V\left[G \cap \mathbb{P}_{\beta}^{n}\right]$ " for every $\beta \in \alpha$, it follows that there must be $G^{\prime}$ containing both $p^{*}$ and $q$ which is $\mathbb{P}_{\alpha}^{n} /\left(G \cap \mathbb{P}_{\beta}^{n}\right)$ generic over $\mathfrak{M}\left[G \cap \mathbb{P}_{\beta}^{n}\right]$ and such that the interpretation of $x$ in $\mathfrak{M}\left[G \cap \mathbb{P}_{\beta}^{n} * G^{\prime}\right]$ is different from the interpretation of $x$ in $\mathfrak{M}[G]$.

\footnotetext{
${ }^{10}$ This definition is somewhat similar to Besicovitch's definition of a contingent which can be found, for example, on page 262 of [7].
} 
Since $\mathcal{G}$ and $P_{1, m}$ are both Polish spaces, it follows directly from an analysis of the definition of the relation $\tau$ that it is an analytic subset of $\mathcal{G} \times P_{1, m}$. It is then possible to appeal to the von Neumann Selection Theorem to find a Baire measurable function $\Delta: \mathcal{G} \rightarrow P_{1, m}$ such that $\Delta(G) \in \tau(G)$ for each $G \in \mathcal{G}$. Let $W \subseteq \mathcal{G}$ be a dense $G_{\delta}$ such that $\Delta$ is continuous on $W$ and let $W_{i}$ be dense open sets such that $W=\bigcap_{i \in \omega} W_{i}$.

The next step will be to construct a fusion sequence $\left\{p_{i}\right\}$ so that the following conditions are satisfied:

- if $h \in \prod_{\gamma \in \Gamma_{i}} f(\gamma)_{n+1}$ (recall that $\Gamma_{i}$ is the domain of $f_{i}$ ), then $\mathcal{U}\left(p_{i}[h]\right) \subseteq W_{i}$;

- if $h_{0}$ and $h_{1}$ are in $\prod_{\gamma \in \Gamma_{i}} f(\gamma)_{n}+1$, then the images $\Psi\left(\mathcal{U}\left(p_{i}\left[h_{0}\right]\right)\right)$ and $\Psi\left(\mathcal{U}\left(p_{i}\left[h_{1}\right]\right)\right)$ have disjoint closures;

- if $G$ belongs to $W, H_{i}(G)=H_{i}\left(G^{\prime}\right)$ and $H_{i+1}(G) \neq H_{i+1}\left(G^{\prime}\right)$, then

$$
\rho\left(\Psi(G)-\Psi\left(G^{\prime}\right), \Delta(G)\right)<1 / i .
$$

First, it will be shown that if such a fusion sequence can be constructed, then the proof is complete. In particular, let $L$ be the image under $\Psi$ of the set of all $G \in \mathcal{G}$ such that $H_{i}(G)$ is defined for all $i \in \omega$. Let $r$ be the fusion of the sequence $\left\{p_{i}\right\}_{i \in \omega}$ and note that $r \Vdash_{\mathbb{P}_{\alpha}^{n}}$ " $x \in L$ ".

To see that $L$ is continuously 1-smooth, suppose that $z=\Psi(G) \in L$ and let $\epsilon>0$. It must first be shown that there is $\delta>0$ such that if $z^{\prime} \in L$ and $\left\|z-z^{\prime}\right\|<\delta$, then $\rho\left(\Delta(G), z-z^{\prime}\right)<\epsilon$. To this end, let $i>1 / \epsilon$ and let $\delta$ be so small that if $h$ and $h^{\prime}$ are distinct functions in $\prod_{\gamma \in \Gamma_{i}}{ }^{f(\gamma)} n+1$, then the distance between $\Psi\left(\mathcal{U}\left(\left(p_{i}[h]\right)\right)\right)$ and $\Psi\left(\mathcal{U}\left(\left(p_{i}\left[h^{\prime}\right]\right)\right)\right)$ is greater than $\delta$. Therefore, if $\left\|z-z^{\prime}\right\|<\delta$, it follows that $H_{i}\left(G^{\prime}\right)=H_{i}(G)$ where $G^{\prime}$ is any member of $W$ such that $\Psi\left(G^{\prime}\right)=z^{\prime}$. Let $j \in \omega$ be the greatest integer such that $H_{j}(G)=H_{j}\left(G^{\prime}\right)$. Since both $G$ and $G^{\prime}$ belong to $W$ and, since $H_{j+1}(G) \neq H_{j+1}\left(G^{\prime}\right)$, it follows that $\rho\left(\Delta(G), z-z^{\prime}\right)=$ $\rho\left(\Delta(G), \Psi(G)-\Psi\left(G^{\prime}\right)\right)<1 / j<1 / i<\epsilon$. Finally, the preceding argument actually established that $\Psi^{-1}$ is continuous on $L$. Hence so is $\Delta \circ \Psi^{-1}$ and this implies that $L$ is not only 1 -smooth but also continuously 1 -smooth.

The next claim will be used in showing that the desired fusion sequence can be constructed. The basic idea is that at stage $i$ of the induction some finite subset $A$ of $\alpha$ on which some fusion has already taken place is given, as well as some ordinal $\beta(i)$ - which comes from the bookkeeping functions $\left\{f_{j}\right\}_{j \in \omega}$ - in this finite set on which some new branching must be frozen. On $A$ finitely many finite subtrees corresponding to the coordinates of the condition $p_{i}$ have already been determined. For each maximal branch through these possibilities a possible branching at coordinate $\beta(i)$ will be frozen. Moreover, these branchings will be chosen in a coherent way so that, in the end, they can all be pasted together without contradiction. Essentially the differences between conditions corresponding to different branches will occur above the maximum of $A$ and this is where the hypothesis that $\alpha$ is a limit ordinal is used. The chief difficulty is that these branchings will differ on the values they assign to the real $x$ and a way must be found to control the angles between them. This is the role of Claim 1. The ordinal $\beta$ in this claim plays the part of $\beta(i)$ and the conditions $q_{i}$ provide the branching at $\beta$. Notice their coherence below $\alpha$ and the fact that the third condition controls the angle between the values determined for $x$. These conditions are then pasted together by following conditions (a) to (g).

Claim 1. Suppose that $\epsilon>0, q \in \mathbb{P}_{\alpha}^{n} \cap \mathfrak{M}, \beta \in \alpha$ and $J \in \omega$. Then there exist conditions $\left\{q_{i}\right\}_{i=0}^{n} \subseteq \mathfrak{M}$ such that 
1. $q_{i} \leq q$ for $i \in n+1$;

2. $q_{i} \uparrow \beta=q_{i^{\prime}} \uparrow \beta$ for $i$ and $i^{\prime}$ in $n+1$;

3. if $\left\{G_{i}\right\}_{i=0}^{n} \subseteq W$ and $q_{i} \in G_{i}$ for each $i \leq n$, then

$$
\rho\left(\Psi\left(G_{i}\right)-\Psi\left(G_{j}\right), \Delta\left(G_{i}\right)\right)<\epsilon
$$

so long as $i \neq j$;

4. $q_{i} \Vdash_{\mathbb{P}_{\alpha}^{n}}$ " $x \in E_{i}$ " for closed balls $E_{i}$ such that $E_{i} \cap E_{i^{\prime}}=\emptyset$ if $i \neq i^{\prime}$;

5. $q_{i}\left\lceil\beta\right.$ decides all the values of $I_{q_{i}(\beta)}\left\lceil J^{J}(n+1)\right.$.

Assuming the claim, suppose that $p_{i}$ of the fusion sequence have been constructed satisfying the induction requirements. Let $\left\{h_{s}\right\}_{s \in k}$ enumerate all the elements of $\prod_{\gamma \in \Gamma_{i}}{ }^{f_{i}(\gamma)}(n+1)$ and let $\mu \in \alpha \cap \mathfrak{M}$ contain the domain of $f_{i+1}$. Let $q_{s}^{0, j}=p_{i}\left[h_{s}\right]$ for each $s \in k$ and $j \leq n$. Proceed by induction on $y \in k$ to construct $q_{s}^{y, j}$ such that

a. $q_{s}^{y, j} \leq q_{s}^{y-1, j}$ for each $y \leq k$;

b. if $\mu^{\prime} \leq \mu$ and $h_{s} \uparrow \mu^{\prime}=h_{s^{\prime}} \uparrow \mu^{\prime}$, then $q_{s}^{y, j}\left\lceil\mu^{\prime}=q_{s^{\prime}}^{y, j}\left\lceil\mu^{\prime}\right.\right.$;

c. $\mathcal{U}\left(q_{y}^{y+1, j}\right) \subseteq W_{i}$ for $y \leq k$ and $j \in n+1$;

d. if $q_{y}^{y+1, j} \in G \in W, q_{y}^{y+1, j^{\prime}} \in G^{\prime} \in W$ and $j \neq j^{\prime}$, then $\rho\left(\Psi(G)-\Psi\left(G^{\prime}\right), \Delta(G)\right)$ $<1 / i$

e. for all $y \leq k$ and $j \leq n$ the condition $q_{y}^{y+1, j}\lceil\beta(i)$ decides the value of $I_{q_{y}^{y+1, j}(\beta(i))}\left(h_{y}(\beta(i)) \wedge j\right)$;

f. $q_{y}^{y+1, j} \vdash_{\mathbb{P}_{\alpha}^{n}}$ " $x \in E_{y}^{j}$ " where $\left\{E_{y}^{j}\right\}_{j=0}^{n}$ is a pairwise disjoint collection of closed balls ;

g. if $y \leq s$, then $q_{s}^{y, j}=q_{s}^{y, j^{\prime}}$ for all $j$ and $j^{\prime}$ in $n+1$.

To see that this induction can be carried out, suppose that $\left\{q_{s}^{y, j}\right\}_{s \in k}^{j \leq n}$ have been defined. From induction hypothesis (g.) it follows that there is some $q^{\prime \prime}$ such that $q^{\prime \prime}=q_{y}^{y, j}$ for all $j \in n+1$. Since $W_{i}$ is dense open, it is possible to find $q^{\prime} \leq q^{\prime \prime}$ such that $\mathcal{U}\left(q^{\prime}\right) \subseteq W_{i}$. Extend $q^{\prime}$ to $q$ such that $q\left\lceil\gamma \Vdash\right.$ " $q(\gamma) \in \mathfrak{S}^{*}(n)$ " for every $\gamma$ in the domain of $f_{i}$ and, furthermore, $q\left\lceil\beta(i)\right.$ decides the value of $I_{q(\beta(i))}\left(h_{y}(\beta(i)) \wedge j\right)$ for each $j \leq n$. Moreover, use the claim to find conditions $\left\{q_{y}^{y+1, j}\right\}_{j \in n+1}$ such that

- $q_{y}^{y+1, j} \leq q$ for $j \in n+1$;

- $q_{y}^{y+1, j}\left\lceil\mu=q_{y}^{y+1, j^{\prime}}\left\lceil\mu\right.\right.$ for $j$ and $j^{\prime}$ in $n+1$;

- if $\left\{G_{j}\right\}_{j \in n+1} \subseteq W$ and $q_{y}^{y+1, j} \in G_{j}$ for each $j \in n+1$, then

$$
\rho\left(\Psi\left(G_{j}\right)-\Psi\left(G_{j^{\prime}}\right), \Delta\left(G_{j}\right)\right)<1 /(i+1)
$$

so long as $j \neq j^{\prime}$;

- $q_{y}^{y+1, j} \Vdash_{\mathbb{P}_{\alpha}^{n}}$ " $x \in E_{y}^{j}$ " for closed balls $E_{y}^{j}$ such that $E_{y}^{j} \cap E_{y}^{j^{\prime}}=\emptyset$ if $j \neq j^{\prime}$;

- $q_{y}^{y+1, j}\left\lceil\beta(i)\right.$ decides the value of $I_{q_{y}^{y+1, j}(\beta(i))}\left(h_{y}(\beta(i)) \wedge j\right)$.

If $s \neq y$, let $b(s)$ be the least member of the domain of $f_{i}$ such that $h_{s}(b(s)) \neq$ $h_{y}(b(s))$ and define $q_{s}^{y+1, j}$ to be the largest lower bound of $q_{y}^{y+1, j}\left\lceil b(s)\right.$ and $q_{s}^{y, j}$. It is easily verified that all of the induction requirements are satisfied.

Now, if $h$ is a member of $\prod_{\gamma \in \Gamma_{i+1}} f_{i+1}(\gamma)(n+1)$, then let $j(h) \leq n$ and $h_{s}$ be a member of $\prod_{\gamma \in \Gamma_{i}}{ }^{f_{i}(\gamma)}(n+1)$ such that $h(\beta(i))=h_{s}(\beta(i)) \wedge j(h)$ and, if $\gamma \neq \beta(i)$, then $h(\gamma)=h_{s}(\gamma)$. Define $p_{i+1}$ to be the join of all the conditions $\left\{q_{s}^{k, j}\right\}$ and observe that $p_{i+1}[h] \leq q_{s}^{k, j(h)}$ and, furthermore, because $p_{i+1}$ is defined as a join,

$$
p_{i+1} \Vdash " I_{p_{i+1}(\gamma)}\left(h(\gamma)\lceil m)=I_{p_{i}(\gamma)}(h(\gamma) \uparrow m) "\right.
$$


for $m \leq f_{i}(\gamma)$. Hence $p_{i+1}$ satisfies the requirements of a fusion sequence. Note that if $y \neq y^{\prime}$, then, by the induction hypothesis on $p_{i}, \Psi\left(\mathcal{U}\left(p_{i}\left[h_{y}\right]\right)\right)$ and $\Psi\left(\mathcal{U}\left(p_{i}\left[h_{y^{\prime}}\right]\right)\right)$ have disjoint closures so it may be assumed that $E_{y}^{j} \cap E_{y^{\prime}}^{j^{\prime}}=\emptyset$. Note that if $H_{i}(G)=H_{i}\left(G^{\prime}\right)$ and $H_{i+1}(G) \neq H_{i+1}\left(G^{\prime}\right)$, then there is some $y \leq k$ and $j \neq j^{\prime}$ such that $q_{y}^{y+1, j} \in G$ and $q_{y}^{y+1, j^{\prime}} \in G^{\prime}$. Hence the third induction requirement follows directly from (d). Thus the three requirements of the desired fusion sequence are satisfied.

All that remains to be done is to prove the claim. To this end, let $q, \epsilon$ and $\beta$ be given. Using the fact that $W$ is dense, let $G^{*} \in W$ be arbitrary such that $q \in G^{*}$. Using the continuity of $\Delta$ on $W$, find $q^{\prime} \in G^{*}$ such that $q^{\prime} \leq q$ and, if $G^{\prime} \in W$ and $q^{\prime} \in G^{\prime}$, then $\rho\left(\Delta\left(G^{*}\right), \Delta\left(G^{\prime}\right)\right)<\epsilon / 2$. Let $p^{-1}=q^{\prime}$. First notice that it suffices to construct by induction on $i$ a sequence $\left\{\left(E_{i}, E^{i}, G_{i}, p_{i}, p^{i}\right)\right\}_{i \in n+1}$ such that $\left\{\left(E_{i}, E^{i}, p_{i}, p^{i}\right)\right\}_{i \in n+1} \in \mathfrak{M}$ and such that for each $i \in n+1$

1. $p_{i} \leq p^{i-1}$,

2. $p^{i} \leq p^{i-1}$,

3. $G_{i} \cap \mathbb{P}_{\beta}^{n}=G^{*} \cap \mathbb{P}_{\beta}^{n}$,

4. $p^{i} \in G^{*}$ for each $i$,

5. $p_{i} \in G_{i}$ for each $i$,

6. $E^{i}$ and $E_{i}$ are disjoint closed subsets of $\mathbb{R}^{m}$,

7. $E^{i+1} \cup E_{i+1} \subseteq E^{i}$,

8. $\Psi\left(\mathcal{U}\left(p^{i}\right)\right)$ is contained in the interior of $E^{i}$,

9. $\Psi\left(\mathcal{U}\left(p_{i}\right)\right)$ is contained in the interior of $E_{i}$,

10. $\rho\left(x-x^{\prime}, \Delta\left(G^{*}\right)\right)<\epsilon / 2$ if $x \in E^{i}$ and $x^{\prime} \in E_{i}$.

The reason this suffices is that, having done so, using conditions (3) and (5) it is possible to find a single $p \in G^{*} \cap \mathbb{P}_{\beta}^{n}$ extending each $p_{i}\lceil\beta$ and such that $p\lceil\beta$ decides all the values of $I_{p(\beta)} \uparrow{ }^{J} n$. Let $q_{i}$ be the greatest lower bound of both $p_{i}$ and $p$. It follows that $q_{i} \leq p_{i} \leq p^{i-1} \leq p^{-1}=q^{\prime} \leq q$. Moreover, $q_{i}\lceil\beta=p$ for each $i \in n+1$. Also, it follows from conditions (6) and (7) that the sets $\left\{E_{i}\right\}_{i \in n+1}$ are pairwise disjoint closed sets and $p_{i} \Vdash$ " $x \in E_{i}$ " by condition (9). Finally, suppose that $i \neq j,\left\{G_{i}, G_{j}\right\} \subseteq W, q_{i} \in G_{i}$ and $q_{j} \in G_{j}$. Then $\rho\left(\Delta\left(G_{i}\right), \Delta\left(G^{*}\right)\right)<\epsilon / 2$ because $q_{i} \leq q^{\prime}$. Hence

$$
\rho\left(\Psi\left(G_{i}\right)-\Psi\left(G_{j}\right), \Delta\left(G_{i}\right)\right)<\epsilon
$$

by condition (10).

To carry out the induction, suppose that $\left\{\left(E_{i}, E^{i}, G_{i}, p_{i}, p^{i}\right)\right\}_{i \in J}$ have been constructed. From the definition of $\Delta\left(G^{*}\right)$ it follows that there is $G_{J}$ such that

- $G_{J} \cap \mathbb{P}_{\beta}^{n}=G^{*} \cap \mathbb{P}_{\beta}^{n}$,

- $p^{J-1} \in G_{J}$,

- $\rho\left(\Psi\left(G^{*}\right)-\Psi\left(G_{J}\right), \Delta\left(G^{*}\right)\right)<\epsilon / 4$,

- $\Psi\left(G^{*}\right) \neq \Psi\left(G_{J}\right)$.

Let $E^{J}$ and $E_{J}$ be disjoint closed neighbourhoods of $\Psi\left(G^{*}\right)$ and $\Psi\left(G_{J}\right)$ respectively such that $\rho\left(x-x^{\prime}, \Delta\left(G^{*}\right)\right)<\epsilon / 2$ for any $x \in E^{J}$ and $x^{\prime} \in E_{J}$. Since induction hypothesis (8) implies that $\Psi\left(\mathcal{U}\left(p^{J-1}\right)\right)$ is contained in the interior of $E^{J-1}$ and $p^{J-1} \in G_{J}$, it follows that it may be assumed that $E^{J} \cup E_{J} \subseteq E^{J-1}$. From the continuity of $\Psi$ it is possible to find $p_{J} \in G_{J}$ and $p^{J} \in G^{*}$ extending $p^{J-1}$ such that $\Psi\left(\mathcal{U}\left(p_{J}\right)\right)$ is contained in the interior of $E_{J}$ and $\Psi\left(\mathcal{U}\left(p^{J}\right)\right)$ is contained in the interior of $E^{J}$. All of the induction hypotheses are now satisfied. 
It is worth noting that nothing very specific to $\mathfrak{S}(n)$ was used in the proof of Lemma 5.1. A similar result can be obtained for almost any partial order which is sufficiently similar to Sacks forcing that a fusion argument, like the one of Lemma 5.1, can be applied to it.

The possibility that $\alpha$ is a successor must now be considered. The proof has the same structure as the limit case but the details are different. In particular, the successor case requires the use of higher dimensional tangent planes and so, unlike the limit case, the use of $\mathfrak{S}(n)$ here will be crucial.

Lemma 5.2. If $\alpha$ is a successor ordinal, then there are a continuously n-smooth set in $\mathbb{R}^{m}, L$, and a condition $r \leq p^{*}$ such that $r \Vdash_{\mathbb{P}_{\alpha}^{n}}$ " $x \in L$ ".

Proof. Let $\alpha=\beta+1$. For each $G \in \mathcal{G}$ let $\tau(G)$ be the set of all $V \in P_{n, m}$ such that for all $\epsilon>0$ and $q \in G$ there is a family $a \in[\mathcal{G}]^{n+1}$ such that

1. $G \in a=\left\{G_{0}, G_{1}, \ldots G_{n}\right\}$,

2. $G_{i} \cap \mathbb{P}_{\beta}^{n}=G \cap \mathbb{P}_{\beta}^{n}$ for each $i \leq n$,

3. $q\left\lceil\beta * q(\beta)\langle\{(0, i)\}\rangle \in G_{i}\right.$ for each $i \leq n$,

4. $\Psi\lceil a$ is one-to-one,

5. $\rho\left(V, \Psi\left(G_{i}\right)-\Psi\left(G_{j}\right)\right)<\epsilon$ for $\{i, j\} \in[n+1]^{2}$.

As in Lemma 5.1, it must be noted that if $G \in \mathcal{G}$, then $\tau(G) \neq \emptyset$. Notice that $\tau(G) \supseteq \bigcap_{q \in G} \overline{\tau(G, q)}$ where $\tau(G, q)$ is defined to be the set of all spaces generated by

$$
\left\{\Psi\left(G^{\prime \prime}\right)-\Psi\left(G^{\prime}\right):\left\{G^{\prime}, G^{\prime \prime}\right\} \in[a]^{2}\right\}
$$

where $a$ satisfies conditions (1) to (4) with respect to $q$. Notice also that its dimension does not exceed $n$ and hence, it belongs to $P_{n, m}$. Since $P_{n, m}$ is compact and $\{\overline{\tau(G, q)}\}_{q \in G}$ has the finite intersection property, it suffices to show that if $q \in G$, then $\tau(G, q) \neq \emptyset$. In $\mathfrak{M}\left[G \cap \mathbb{P}_{\beta}^{n}\right]$ the name $q(\beta)$ is interpreted as a condition in $\mathfrak{S}(n)$. It follows that in $\mathfrak{M}\left[G \cap \mathbb{P}_{\beta}^{n}\right]$ there must be $q^{\prime} \leq q \uparrow \beta$ which decides the root of $q(\beta)$ to be $r$. Let $q_{i}=q^{\prime} \uparrow \beta * q(\beta)\langle\{(0, i)\}\rangle$. Since

$$
p^{*} \vdash_{\mathbb{P}_{\alpha}^{n}} \text { " } x \notin \mathfrak{M}\left[G \cap \mathbb{P}_{\beta}^{n}\right] ",
$$

it is an easy matter to choose $G_{i}^{\prime}$ which is $\mathfrak{S}(n)$ generic over $\mathfrak{M}\left[G \cap \mathbb{P}_{\beta}^{n}\right], q_{i} \in G_{i}^{\prime}$ and such that the interpretation of $x$ in $\mathfrak{M}\left[G \cap \mathbb{P}_{\beta}^{n} * G_{i}^{\prime}\right]$ is different from the interpretation of $x$ in $\mathfrak{M}\left[G \cap \mathbb{P}_{\beta}^{n} * G_{j}^{\prime}\right]$ unless $i=j$. It follows that, letting $G_{i}=G \cap \mathbb{P}_{\beta}^{n} * G_{i}^{\prime}$, the space generated by

$$
\left\{\Psi(G)-\Psi\left(G_{i}\right)\right\}_{i=1}^{n}
$$

belongs to $\tau(G, q)$.

As in Lemma 5.1, it is possible to find a Baire measurable function $\Delta: \mathcal{G} \rightarrow P_{n, m}$ such that $\Delta(G) \in \tau(G)$ for each $G \in \mathcal{G}$. Let $W$ be a dense $G_{\delta}$ on which $\Delta$ is continuous. Let $W_{n}$ be dense open sets such that $W=\bigcap_{i \in \omega} W_{n}$.

As before, the next step will be to construct a fusion sequence $\left\{p_{i}\right\}_{i \in \omega}$ so that the following conditions are satisfied:

- if $h$ is in $\prod_{\gamma \in \Gamma_{i}} f(\gamma) n+1$, then $\mathcal{U}\left(p_{i}[h]\right) \subseteq W_{i}$,

- if $h_{0}$ and $h_{1}$ are distinct elements of $\prod_{\gamma \in \Gamma_{i}} f(\gamma)_{n}+1$, then the images

$$
\Psi\left(\mathcal{U}\left(p_{i}\left[h_{0}\right]\right)\right) \text { and } \Psi\left(\mathcal{U}\left(p_{i}\left[h_{1}\right]\right)\right)
$$

have disjoint closures; 
- if $G$ belongs to $W, H_{i}(G)=H_{i}\left(G^{\prime}\right)$ and $H_{i+1}(G) \neq H_{i+1}\left(G^{\prime}\right)$, then

$$
\rho\left(\Psi(G)-\Psi\left(G^{\prime}\right), \Delta(G)\right)<1 / i .
$$

Observe that if such a fusion sequence can be constructed, then the proof is complete. In particular, let $L$ be the image under $\Psi$ of the set of all $G \in \mathcal{G}$ such that $H_{i}(G)$ is defined for all $i \in \omega$. Let $r$ be the fusion of the sequence $\left\{p_{i}\right\}_{i \in \omega}$. Then $r \Vdash$ " $x \in L$ ". The proof that $L$ is continuously $n$-smooth is exactly the same as in Lemma 5.1.

To see that the fusion sequence can be found, suppose that $p_{i}$ has been constructed satisfying the induction requirements. Let $\left\{h_{s}\right\}_{s \in k}$ enumerate

$$
\prod_{\gamma \in \Gamma_{i}} f_{i}(\gamma)(n+1)
$$

and let $q_{s}^{0, j}=p_{i}\left[A\left(h_{s}\right)\right]$ for each $s \in k$. Proceed by induction on $y$ to construct $q_{s}^{y, j}$ such that

- $q_{s}^{y, j} \leq q_{s}^{y-1, j}$ for each $y \leq k$;

- if $\mu \leq \alpha$ and $h_{s} \uparrow \mu=h_{s^{\prime}}\left\lceil\mu\right.$, then $q_{s}^{y, j}\left\lceil\mu=q_{s^{\prime}}^{y, j}\lceil\mu\right.$;

- $\mathcal{U}\left(q_{y}^{y+1, j}\right) \subseteq W_{i}$ for $y \leq k$ and $j \in n+1$;

- if $q_{y}^{y+1, j} \in G \in W, q_{y}^{y+1, j^{\prime}} \in G^{\prime} \in W$ and $j \neq j^{\prime}$, then $\rho\left(\Psi(G)-\Psi\left(G^{\prime}\right), \Delta(G)\right)$ $<1 / i$

- for all $y \leq k$ and $j \leq n$ the condition $q_{y}^{y+1, j} \uparrow \beta(i)$ decides the value of $I_{q_{y}^{y+1, j}(\beta(i))}\left(h_{y}(\beta(i)) \wedge j\right)$

- $q_{y}^{y+1, j} \Vdash_{\mathbb{P}_{\alpha}^{n}}$ " $x \in E_{y}^{j}$ " where $\left\{E_{y}^{j}\right\}_{j \in n+1}$ is a pairwise disjoint collection of closed balls;

- if $y \leq s$, then $q_{s}^{y, j}=q_{s}^{y, j^{\prime}}$ for all $j$ and $j^{\prime}$ in $n+1$.

To see that this induction can be carried out, suppose that $\left\{q_{s}^{y, j}\right\}_{s \in k}$ have been defined. From the last induction hypothesis it follows that there is some $q^{\prime \prime}$ such that $q^{\prime \prime}=q_{y}^{y, j}$ for all $j \in n+1$. Since $W_{i}$ is dense open, it is possible to find $q^{\prime} \leq q^{\prime \prime}$ such that $\mathcal{U}\left(q^{\prime}\right) \subseteq W_{i}$. Extend $q^{\prime}$ to $q$ such that $q\lceil\beta(i)$ decides the value of $I_{q(\beta(i))}\left(h_{y}(\beta(i)) \wedge j\right)$ for each $j \leq n$.

Using the fact that $W$ is dense in $\mathcal{G}$, let $G \in W$ be such that $q \in G$. Using the definition of $\tau(G)$, let $a \in[\mathcal{G}]^{n+1}$ be such that Conditions 1 to 5 hold. It follows from Condition 3 that $\hat{q}_{j}=q\left\lceil\beta * q(\beta)\langle\{(0, j)\}\rangle \in G_{j}\right.$ for each $j \leq n$. Using Condition 5, the continuity of $\Delta$ and $\Psi$ as well as the fact that $p^{*} \vdash_{\mathbb{P}_{\alpha}^{n}}$ " $x \notin \mathfrak{M}\left[G \cap \mathbb{P}_{\beta}^{n}\right]$ " it is possible to extend each $\hat{q}_{j}$ to $q_{y}^{y+1, j}$ such that

- $q_{y}^{y+1, j} \leq q$ for $j \in n+1$;

- $q_{y}^{y+1, j}\left\lceil\beta=q_{y}^{y+1, j^{\prime}}\left\lceil\beta\right.\right.$ for $j$ and $j^{\prime}$ in $n+1$;

- if $\left\{G_{j}\right\}_{j \in n+1} \subseteq W$ and $q_{y}^{y+1, j} \in G_{j}$ for each $j \in n+1$, then

$$
\rho\left(\Psi\left(G_{j}\right)-\Psi\left(G_{j^{\prime}}\right), \Delta\left(G_{j}\right)\right)<1 /(i+1)
$$

so long as $j \neq j^{\prime}$;

- $q_{y}^{y+1, j} \Vdash_{\mathbb{P}_{\alpha}^{n}}$ " $x \in E_{y}^{j}$ " for closed balls $E_{y}^{j}$ such that $E_{y}^{j} \cap E_{y}^{j^{\prime}}=\emptyset$ if $j \neq j^{\prime}$.

Note that guaranteeing that the second condition is satisfied is easy since $\hat{q}_{j} \in G_{j}$ for each $j \leq n$ and, hence, are all compatible. If $s \neq y$, let $b(s)$ be the least member of the domain of $f_{i}$ such that $h_{s}(b(s)) \neq h_{y}(b(s))$ and define $q_{s}^{y+1, j}$ to be the least upper bound of $q_{y}^{y+1, j}\left\lceil b(s)\right.$ and $q_{s}^{y, j}$. It is easily verified that all of the induction requirements are satisfied. Define $p_{i+1}$ to be the join of all the conditions $q_{s}^{k, j}$. This 
satisfies the three requirements of the desired fusion sequence regardless of whether $\beta=\beta(i)$.

Lemmas 5.2 and 5.1 together show that the ground model continuously $n$-smooth sets are sufficient to cover all reals added by iteratively adding $\omega_{2}$ reals with the partial order $\mathfrak{S}(n)$. Combined with Corollary 4.1, this immediately gives the following theorem.

Theorem 5.1. If $1 \leq n<m \in \omega$, then it is consistent, relative to the consistency of set theory itself, that $\operatorname{cov}\left(\mathcal{D}_{m, n-1}\right)=\aleph_{2}$ and $\operatorname{cov}\left(\mathcal{C}_{m, n}^{1}\right)=\aleph_{1}$.

Corollary 5.1. It is consistent, relative to the consistency of set theory itself, that $\mathfrak{s}_{n-1}=\aleph_{2}$ and $\mathfrak{s}_{n}=\aleph_{1}$ for $n>1$.

Proof. The model is the same as that for Theorem 5.1. Since $\mathfrak{s}_{n-1} \geq \operatorname{cov}\left(\mathcal{D}_{m, n}\right)$, it follows that $\mathfrak{s}_{n-1}=\omega_{2}$. Since the standard Sacks real argument shows that each partial order $\mathfrak{S}(n)$ is $\omega_{\omega}$-bounding, it follows from the preservation of this property by countable support iteration [8] that this model also satisfies that $\mathfrak{d}=\aleph_{1}$. It is shown in Proposition 3.1 that $\omega_{1} \leq \mathfrak{d}_{n} \leq \mathfrak{d}$. Hence, $\mathfrak{d}_{n}=\mathfrak{d}=\omega_{1}$ in this model. From Corollary 3.3 and the fact that $\operatorname{cov}\left(\mathcal{C}_{n+1, n}^{1}\right)=\mathfrak{d}$ in this model it follows that, in this model, the equality $\operatorname{cov}\left(\mathcal{C}_{n+1, n}^{1}\right)=\operatorname{cov}\left(\mathcal{S}_{n+1, n}\right)=\mathfrak{s}_{n}$ must hold. Hence $\mathfrak{s}_{n}<\mathfrak{s}_{n-1}$ in this model.

\section{Decomposing continuous Functions}

In [6] the authors consider the following question: If $\mathcal{A}$ and $\mathcal{B}$ are two families of functions between Polish spaces, what is the least cardinal $\kappa$ such that every member of $\mathcal{A}$ can be decomposed into $\kappa$ members of $\mathcal{B}$ - this cardinal $\kappa$ the authors call $\operatorname{dec}(\mathcal{A}, \mathcal{B})$. The most natural class to consider for $\mathcal{B}$ is the class of continuous functions and the problem to which it gives rise had been posed by Lusin, who wondered whether every Borel function could be decomposed in countably many continuous functions. In more recent times, it has been shown by Abraham, Rubin and Shelah to be consistent that every monotone function of size less than $\mathfrak{c}$ can be decomposed into countably many continuous functions [1]. Various results concerning $\operatorname{dec}\left(\mathcal{B}_{1}, \mathcal{C}\right)$ where $\mathcal{B}_{1}$ is the class of pointwise limits of continuous functions and $\mathcal{C}$ is the class of continuous functions can be found in [9],[11] and [10].

The question of determining $\operatorname{dec}(\mathcal{C}, \mathcal{D})$ where $\mathcal{D}$ is the class of functions which are differentiable on their domain was raised by M. Morayne and J. Cichon. It was not known whether it is consistent that $\operatorname{dec}(\mathcal{C}, \mathcal{D})<2^{\aleph_{0}}$ and the best lower bound for $\operatorname{dec}(\mathcal{C}, \mathcal{D})$ has been noted by Morayne to be the additivity of the null ideal. The following result shows that $\operatorname{dec}(\mathcal{C}, \mathcal{D})<2^{\aleph_{0}}$ is indeed consistent.

Proposition 6.1. $\operatorname{dec}(\mathcal{C}, \mathcal{D}) \leq \operatorname{cov}\left(\mathcal{D}_{2,1}\right)$.

Proof. To show that $\operatorname{dec}(\mathcal{C}, \mathcal{D}) \leq \operatorname{cov}\left(\mathcal{D}_{2,1}\right)$ let $\kappa=\operatorname{cov}\left(\mathcal{D}_{2,1}\right)$ and let $\left\{S_{\eta}\right\}_{\eta \in \kappa}$ be a decomposition of $\mathbb{R}^{2}$ into 1 -smooth sets. Given a continuous function $f: \mathbb{R} \rightarrow \mathbb{R}$, let $f_{\eta}=f \cap S_{\eta}$.

To see that $f_{\eta}$ is differentiable on its domain, let $x$ be in the domain of $f_{\eta}$. Let $m$ be the slope of the tangent line to $S_{\eta}$ at the point $\left(x, f_{\eta}(x)\right)$ allowing the possibility that $m=\infty$. Then, using the fact that $f$ is continuous, it is easily verified that $f_{\eta}^{\prime}(x)=m$. 
It is not known whether the inequality in Proposition 6.1 can be replaced by an equality. If the answer is yes, then this would provide a better lower bound for $\operatorname{dec}(\mathcal{C}, \mathcal{D})$, namely $\operatorname{dec}(\mathcal{C}, \mathcal{D})^{+} \geq 2^{\aleph_{0}}$. However, it can be shown that there is a perfect set $A \subseteq \mathbb{R}$ such that $A \times A$ can be covered by $\operatorname{dec}(\mathcal{C}, \mathcal{D}) 1$-smooth sets. Simply let $A$ be a perfect set such that there is $\theta \in(0, \pi / 4)$ be such that if $a$ and $a^{\prime}$ are distinct points in $A \times A$, then the angle formed by the horizontal axis and the line connecting $a$ and $a^{\prime}$ is different from $\theta$. Let $H_{\theta}$ be the function which projects $\mathbb{R}^{2}$ to the vertical axis along the line at angle $\theta$ with respect to the horizontal axis. Let $H_{\pi / 2}: \mathbb{R} \rightarrow \mathbb{R}$ be the orthogonal projection onto the horizontal axis. Since $H_{\theta}\left\lceil(A \times A)\right.$ is one-to-one and $A \times A$ is compact, it follows that $H_{\theta}^{-1}$ is continuous and, hence, so is $H=H_{\pi / 2} \circ H_{\theta}^{-1}$. Since the domain of $H$ is compact, it can be extended to a continuous function on the entire real line.

Now suppose that $\left\{X_{\eta}\right\}_{\eta \in \kappa}$ are subsets of $\mathbb{R}$ such that $H\left\lceil X_{\eta}\right.$ is differentiable for each $\eta \in \kappa$. To see that $H_{\theta}^{-1}\left(X_{\eta}\right)$ is a 1 -smooth set, suppose that $(x, y) \in H_{\theta}^{-1}\left(X_{\eta}\right)$ is a point at which $H_{\theta}^{-1}\left(X_{\eta}\right)$ does not have a 1-dimensional tangent. In this case it is possible to find distinct slopes $m_{0}$ and $m_{1}$ and sequences $\left\{\left(x_{n}^{i}, y_{n}^{i}\right)\right\}_{n \in \omega}$ for each $i \in 2$ such that

$$
\lim _{n \rightarrow \infty} \frac{y-y_{n}^{i}}{x-x_{n}^{i}}=m_{i}
$$

allowing the possibility of an infinite limit. Let $w_{n}^{i}=H_{\theta}^{-1}\left(x_{n}^{i}, y_{n}^{i}\right)$ and $w=$ $H_{\theta}^{-1}(x, y)$. It follows from the linearity of $H_{\theta}$ that there are distinct $M_{0}$ and $M_{1}$ such that

$$
\lim _{n \rightarrow \infty} \frac{x-x_{n}^{i}}{w-w_{n}^{i}}=M_{i}
$$

and this contradicts the differentiability of $H$ at $w$ since $H\left(w_{n}^{i}\right)=x_{n}^{i}$ and $H(w)=x$.

It has already been remarked that $\operatorname{cov}\left(\mathcal{D}_{2,1}\right) \geq \operatorname{cov}(\mathcal{N})$. This will follow immediately from Proposition 6.1 and the following theorem of M. Morayne.

Theorem 6.1. There is a continuous function from $\mathbb{R}$ to $\mathbb{R}$ which cannot be decomposed into fewer than $\operatorname{cov}(\mathcal{N})$ differentiable functions.

Proof. Let $F: \mathbb{R} \rightarrow \mathbb{R}$ be a continuous function ${ }^{11}$ such that there is a set $A$ of positive measure such that $\left|F^{-1}\{a\}\right|=2^{\aleph_{0}}$ for every $a \in A$. If $\kappa<\operatorname{cov}(\mathcal{N})$ and $\left\{Z_{\zeta}\right\}_{\zeta \in \kappa}$ are subsets of $\mathbb{R}$, let $Z_{\zeta}^{\prime}=\left\{z \in Z_{\zeta}:\left|F^{-1}\{F(z)\}\right| \geq \aleph_{0}\right\}$, then $\bigcup_{\zeta \in \kappa} F\left(Z_{\zeta}^{\prime}\right) \supseteq A$. It is therefore possible to choose $\zeta \in \kappa$ such that the measure of $F\left(Z_{\zeta}^{\prime}\right)$ is positive.

The following theorem of Banach can be found on page 278 of [7]: If $X \subseteq \mathbb{R}$ and $H: X \rightarrow \mathbb{R}$ is continuous, then the set of points in $\mathbb{R}$ whose preimage under $H$ is infinite is of measure zero if and only if the set of values attained by $H$ at points at which $H$ is not differentiable has measure zero. This immediately implies that $F\left\lceil Z_{\zeta}^{\prime}\right.$ is not differentiable at some point.

\footnotetext{
${ }^{11}$ To find such a function let $\pi: 2^{\omega} \times 2^{\omega}$ be the projection function, let $C$ be a Cantor set of positive measure, let $f_{1}: C \rightarrow 2^{\omega}$ and $f_{2}: C \rightarrow 2^{\omega} \times 2^{\omega}$ be homeomorphisms. Let $f=f_{1}^{-1} \circ \pi \circ f_{2}$ and let $F \supseteq f$ be an arbitrary continuous extension of $f$.
} 


\section{REMARKS AND OPEN QUESTIONS}

An interesting by-product of the results established in this paper has to do with the difficulties encountered when iterating with countable support beyond $\omega_{2}$. In this regard, it is interesting to note a consequence of Corollary 3.2 and Theorem 5.1. Since $\mathfrak{S}(1)$ is simply ordinary Sacks forcing, it follows from Lemma 5.2 and Lemma 5.1 that in the model obtained by iteratively adding $\omega_{2}$ Sacks reals to a model of $2^{\aleph_{0}}=\aleph_{1}$ one obtains a model where $\operatorname{cov}\left(\mathcal{D}_{2,1}\right)=\aleph_{1}$. However, by forcing with the product of $\aleph_{3}$ Sacks reals over this same model, one obtains a model where $2^{\aleph_{0}}=\aleph_{3}$ and hence, by Corollary 3.2 , it follows that $\operatorname{cov}\left(\mathcal{D}_{2,1}\right) \geq \aleph_{2}$. A standard reflection argument yields that in some intermediate model, obtained by forcing with the product of only $\aleph_{2}$ Sacks reals, it must be that $\operatorname{cov}\left(\mathcal{D}_{2,1}\right)=$ $\aleph_{2}$. Homogeneity yields that forcing with the product of $\aleph_{2}$ Sacks reals increases $\operatorname{cov}\left(\mathcal{D}_{2,1}\right)$, whereas the iteration does not. This yields an answer to a question of Todorcevic who asked for a simple cardinal invariant to distinguish between the iteration and product of $\omega_{2}$ Sacks reals. It also shows that there is no way of extending the iteration of Sacks reals to obtain a model where $2^{\aleph_{0}}>\aleph_{2}$ and so that most of the reasonable properties of the usual iteration still hold - reasonable here means, of course, that which was used in the preceding argument. The following are some natural questions which require overcoming this difficulty.

Question 7.1. Does the inequality $\operatorname{cov}\left(\mathcal{D}_{2,1}\right)>\aleph_{1}$ imply that $\operatorname{cov}\left(\mathcal{D}_{2,1}\right)=2^{\aleph_{0}}$ ?

Similar questions can be asked about the ideals $\mathcal{S}_{2,1}$ and $\mathcal{C}_{2,1}^{1}$. The same is true of the following question.

Question 7.2. Is it consistent that

$$
\operatorname{cov}\left(\mathcal{D}_{n, m}\right)>\operatorname{cov}\left(\mathcal{D}_{n^{\prime}, m^{\prime}}\right)>\operatorname{cov}\left(\mathcal{D}_{n^{\prime \prime}, m^{\prime \prime}}\right)
$$

for some choice of integers?

Other types of decompositions of the plane may also be of interest. By a curve, will always be meant a one-to-one function. Let $\mathcal{R}$ be the $\sigma$-ideal generated by rectifiable curves in the plane, $\mathcal{L}$ the $\sigma$-ideal generated by Lipschitz curves in the plane and $\mathcal{C}$ be the $\sigma$-ideal generated by continuous curves in the plane. Since $\mathcal{S}_{2,1} \subseteq \mathcal{C} \cap \mathcal{L} \cap \mathcal{R}$, it follows that $\operatorname{cov}(\mathcal{X}) \leq \operatorname{cov}\left(\mathcal{S}_{2,1}\right)$ if $\mathcal{X} \in\{\mathcal{R}, \mathcal{L}, \mathcal{C}\}$. What else can be said about these ideals and their associated invariants?

It has already been remarked in Section 3 that under various assumptions $\operatorname{cov}\left(\mathcal{C}_{m, n}^{1}\right)=\mathfrak{s}_{n}$.

Question 7.3. Is it consistent that $\operatorname{cov}\left(\mathcal{C}_{m, n}^{1}\right) \neq \mathfrak{s}_{n}$ ?

Question 7.4. Is it consistent that $\operatorname{cov}\left(\mathcal{D}_{m, n}\right) \neq \operatorname{cov}\left(\mathcal{D}_{m^{\prime}, n}\right)$ for some $m, m^{\prime}$ and $n ?$

Finally, one can define related cardinal invariants by defining a set to be $(n, m)$ smooth if the number of $m$-dimensional tangent planes at each point is no more than $n$. In effect, this allows corners because, for example, the graph of the absolute value function in $\mathbb{R}^{2}$ is $(2,1)$-smooth. One can then define the associated ideals and consider their cardinal invariants as before. What can be said about these? For example if the plane is covered by $\lambda(7,1)$-smooth sets, is it also covered by $\lambda$ $(6,1)$-smooth sets? 


\section{REFERENCES}

1. A. Abraham, M. Rubin, and S. Shelah. On the consistency of some partition theorems for continuous colorings, and structure of $\aleph_{1}$-dense real order types. Ann. Pure Appl. Logic, 29:123-206, 1985. MR 87d:03132

2. V. Aversa, M. Laczkovich, and D. Preiss. Extension of differentiable functions. Comment. Math. Univ. Carolin., 26(3):597-609, 1985. MR 87c:26022

3. J. E. Baumgartner and R. Laver. Iterated perfect set forcing. Ann. Math. Logic, 17:271-288, 1979. MR 81a:03050

4. A. S. Besicovitch. On tangents to general sets of points. Fund. Math., 22:49-53, 1934.

5. J. Cichon and M. Morayne. Universal functions and generalized classes of functions. Proc. Amer. Math. Soc., 102:83-89, 1988. MR 89c:26003

6. J. Cichoń, M. Morayne, J. Pawlikowski, and S. Solecki. Decomposing Baire functions. J. Symbolic Logic, 56(4):1273-1283, 1991. MR 92j:04001

7. S. Saks. Theory of the Integral. Hafner, New York, 1937.

8. S. Shelah. Proper Forcing, volume 940 of Lecture Notes in Mathematics. Springer-Verlag, Berlin, 1982. MR 84h:03002

9. S. Shelah and J. Steprāns. Decomposing Baire class 1 functions into continuous functions. Fund. Math., 145:171-180, 1994. MR 95i:03110

10. S. Solecki. Decomposing Borel sets and functions and the structure of Baire class 1 functions. J. Amer. Math. Soc. 11:521-550, 1998. CMP 98:13

11. J. Steprāns. A very discontinuous Borel function. J. Symbolic Logic, 58:1268-1283, 1993. MR 95c:03120

Department of Mathematics, York University, 4700 Keele Street, North York, OnTARIO, CANADA, M3J 1P3

E-mail address: steprans@mathstat.yorku.ca 